\title{
Direct interaction of whole-inactivated influenza $A$ and pneumococcal vaccines enhances influenza-specific immunity
}

\author{
Shannon C. David', Todd Norton1, Timona Tyllis 1, Jasmine J. Wilson', Eve V. Singleton', Zoe Laan1, \\ Justin Davies², Timothy R. Hirst ${ }^{1,3,4}$, lain Comerford', Shaun R. McColl', James C. Paton ${ }^{1,4,5}$ and \\ Mohammed Alsharifi $\mathbb{B D}^{1,3,4,5 \star}$
}

The upper respiratory tract is continuously exposed to a vast array of potentially pathogenic viruses and bacteria. Influenza A virus (IAV) has particular synergism with the commensal bacterium Streptococcus pneumoniae in this niche, and co-infection exacerbates pathogenicity and causes significant mortality. However, it is not known whether this synergism is associated with a direct interaction between the two pathogens. We have previously reported that co-administration of a whole-inactivated IAV vaccine $(\gamma$-Flu) with a whole-inactivated pneumococcal vaccine $(\gamma$-PN) enhances pneumococcal-specific responses. In this study, we show that mucosal co-administration of $\gamma$-Flu and $\gamma$-PN similarly augments IAV-specific immunity, particularly tissueresident memory cell responses in the lung. In addition, our in vitro analysis revealed that $S$. pneumoniae directly interacts with both $\gamma$-Flu and with live IAV, facilitating increased uptake by macrophages as well as increased infection of epithelial cells by IAV. These observations provide an additional explanation for the synergistic pathogenicity of IAV and S. pneumoniae, as well as heralding the prospect of exploiting the phenomenon to develop better vaccine strategies for both pathogens.

$\mathrm{C}$ onventional views of pathogen dynamics have rarely deviated from the consideration of single pathogens acting independently of one another. However, pathogen interactions are increasingly recognized as critical contributors to both health and disease progression ${ }^{1-4}$. Such interactions are prevalent at sites harbouring complex populations of commensal and pathogenic microbes, including the gut and respiratory tract. The synergy between the respiratory pathogens IAV and S. pneumoniae is well documented in this setting ${ }^{5,6}$, and leads to exacerbated disease and high mortality $5,7,8$.

Despite the availability of vaccines for both IAV and S. pneumoniae, co-infection is a recurring health issue. Studies have attempted simultaneous administration of existing IAV and pneumococcal vaccines to improve coverage, and while successful at reducing disease incidence (particularly in elderly populations ${ }^{9,10}$ ), this strategy does not offer broad-spectrum protection. Irrespective of co-administration, current vaccines induce vaccine-specific immunity alone and offer minimal protection against emerging isolates due to lack of cross-reactivity. Consequently, inactivated IAV vaccines require annual reformulation to ensure coverage of seasonal strains. The time required to generate these strain-specific vaccines is approximately six months ${ }^{11}$, which is too long in the event of a sudden pandemic to protect against the first wave of infection. The increasing probability of an avian influenza pandemic paired with the increasing prevalence of non-vaccine pneumococcal serotypes underscores the urgent need for new broad-spectrum vaccines.

We have previously published an approach to meet this need using gamma $(\gamma)$-irradiated vaccines comprising whole-inactivated IAV $(\gamma$-Flu $)$ and whole-inactivated pneumococci $(\gamma$-PN).
Intranasal vaccination with $\gamma$-Flu (based on H1N1 or H3N2) confers significant protection against homotypic and heterosubtypic IAV challenges, including avian $\mathrm{H} 5 \mathrm{~N} 1^{12,13}$. We have also shown that $\gamma$-Flu can be exposed to high doses of $\gamma$-irradiation (50kGy) while retaining high-level immunogenicity ${ }^{14}$, enabling $\gamma$-Flu to include pandemic influenza strains. Furthermore, we have reported that mucosal co-administration of $\gamma$-Flu and $\gamma$-PN provides significant protection against lethal co-infection, and results in superior induction of pneumococcal-specific responses ${ }^{15}$. In the current study, we show that $\gamma$-Flu-induced responses are similarly enhanced by co-administration, and demonstrate a direct interaction between both live and inactivated IAV and S. pneumoniae vaccine components, which appears to facilitate increased viral uptake by antigenpresenting cells.

\section{Results}

Co-administration of $\gamma$-irradiated S. pneumoniae with $\gamma$-Flu enhances IAV-specific protection. Our previous work has demonstrated that co-administering $\gamma$-Flu with a previous version of our irradiated pneumococcal vaccine $(\gamma-\mathrm{PN})$ does not adversely affect homotypic protection ${ }^{15}$. We have recently generated a derivative of $\gamma$-PN with enhanced safety and immunogenicity by introducing an attenuating deletion in the $p s a A$ gene (termed $\gamma-\mathrm{PN}(\Delta \mathrm{PsaA})$ ) (S.C.D., Z.L., V. Minhas, A. Chen, J.D., T.R.H., S.R.M., M.A. and J.C.P., manuscript under review). Here, we show that co-administering $\gamma$-Flu with $\gamma$-PN $(\Delta \mathrm{PsaA})$ confers complete protection against homotypic H1N1 (Fig. 1a), whereas PBS-mock-treated and $\gamma$-PN $(\Delta$ PsaA $)$-vaccinated controls were euthanized due to excess weight loss. We then investigated whether co-vaccination

'Research Centre for Infectious Diseases, and Department of Molecular and Biomedical Science, School of Biological Sciences, University of Adelaide, Adelaide, South Australia, Australia. ${ }^{2}$ Australian Nuclear Science and Technology Organisation, Sydney, New South Wales, Australia. ${ }^{3}$ Gamma Vaccines Pty Ltd, Yarralumla, Australian Capital Territory, Australia. ${ }^{4} \mathrm{GPN}$ Vaccines Pty Ltd, Yarralumla, Australian Capital Territory, Australia. ${ }^{5}$ These authors jointly supervised this work: James C. Paton, Mohammed Alsharifi. ^e-mail: mohammed.alsharifi@adelaide.edu.au 

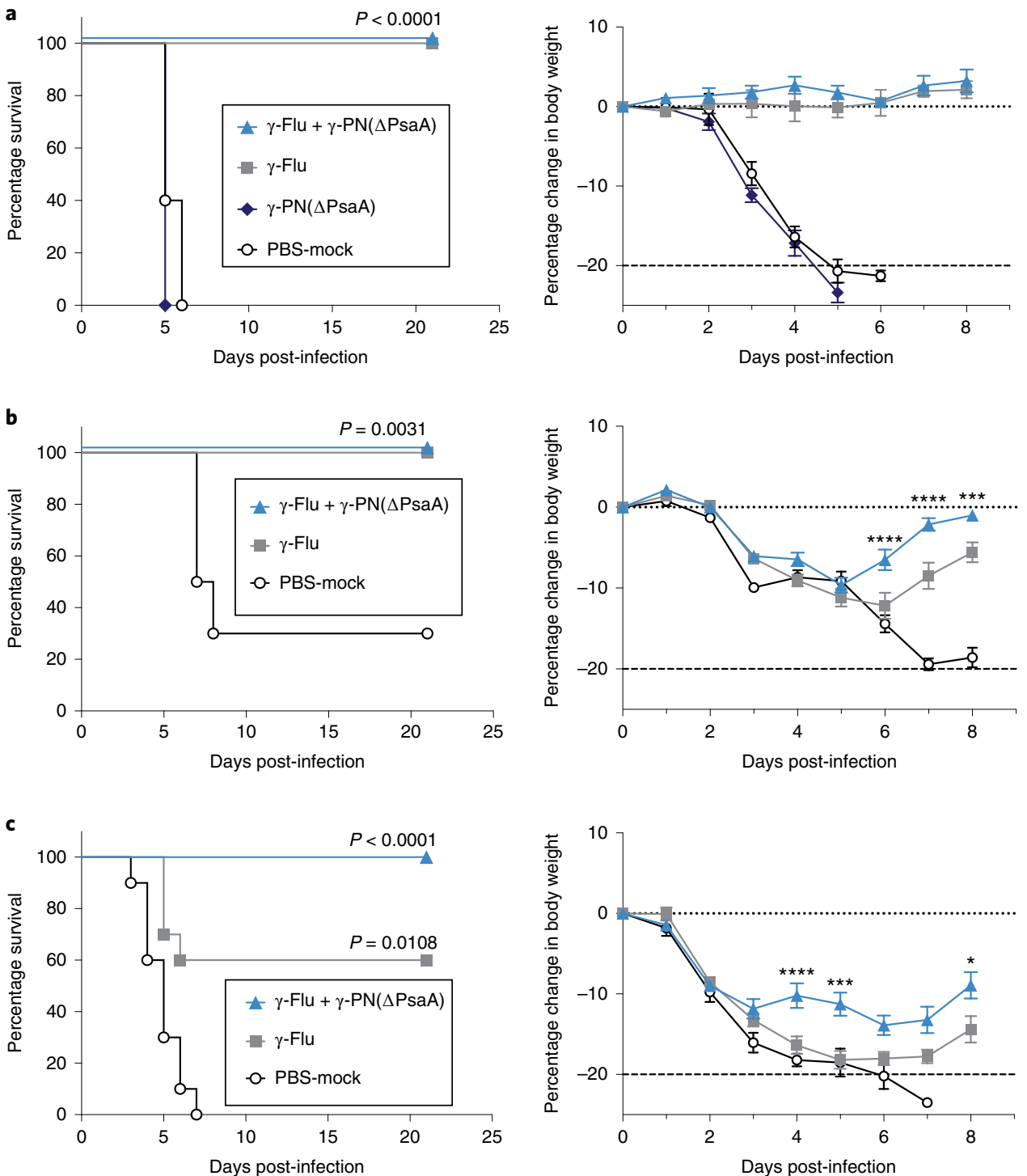

Fig. 1 | Enhanced protection against drifted and heterosubtypic IAV challenge following co-vaccination with $\gamma$-Flu $+\gamma$-PN $(\Delta P \operatorname{PaA})$. Mice were vaccinated i.n. with $\gamma$-Flu alone or co-vaccinated with $\gamma$-Flu $+\gamma$-PN $(\Delta \mathrm{PsaA})$. Control mice were PBS-mock-vaccinated. a-c, At 21 days post-vaccination, mice were challenged i.n. with homotypic H1N1 (A/Puerto Rico/8/34) (a), drifted pdmH1N1 (A/California/07/2009) (b) or heterosubtypic H3N2 (A/ PortChalmers/1/73) (c). Mice were monitored for three weeks for development of clinical symptoms, and euthanized if the total weight loss reached $20 \%$ of the starting weight (dashed line). The data are presented as survival percentages (left panels) and weight loss (mean \pm s.e.m., $n=10$ mice per group) (right panels). The data are compiled from two independent experiments, and were analysed by Fisher's exact test (two-tailed) compared to the PBS-mock control group (left panels) or two-way ANOVA with Tukey's multiple comparisons test $\left({ }^{\star} P<0.05,{ }^{\star \star \star} P<0.001,{ }^{\star \star \star \star} P<0.0001\right.$ compared to the $\gamma$-Flu alone group) (right panels).

would affect $\gamma$-Flu-mediated cross-protection against lethal challenge with drifted pandemic H1N1 (pdmH1N1) or heterosubtypic H3N2 IAV. Figure 1b shows that all mice vaccinated with $\gamma$-Flu or $\gamma$-Flu $+\gamma$-PN $(\Delta \mathrm{PsaA})$ survived lethal challenge with pdmH1N1, with limited weight loss before recovery. This weight loss was expected considering the role of $\mathrm{CD}^{+} \mathrm{T}$ cells in $\gamma$-Flu-mediated protection, and the high, lethal dose used to challenge animals. Interestingly, co-vaccinated mice showed a faster rate of recovery, with significantly reduced weight loss from day 6 onwards compared to mice vaccinated with $\gamma$-Flu alone. To further assess differences in vaccine performance, we challenged animals with an intentionally high dose of heterosubtypic H3N2. Animals vaccinated with $\gamma$-Flu alone showed only $60 \%$ protection, in contrast to the $100 \%$ protection conferred by co-administration of $\gamma$-Flu with $\gamma$-PN( $\Delta$ PsaA) (Fig. 1c). This cross-protection was also associated with significantly reduced weight loss and early recovery when compared to mice receiving $\gamma$-Flu alone.

Co-vaccination does not affect neutralizing antibody responses. To address potential mechanisms underlying the enhanced crossprotection observed for co-vaccinated animals, both humoral and cell-mediated immune responses were investigated. Initially, the functionality of vaccine-induced antibodies was assessed using in vitro neutralization assays. Serum samples from $\gamma$-Flu-vaccinated and co-vaccinated mice were incubated with a fixed titre of live H1N1 (homotypic), before infection of Madin-Darby Canine Kidney (MDCK) monolayers. Complete inhibition of infection was observed following treatment with low serum dilutions for both vaccine groups, while infectivity was detected for samples treated with more diluted sera $(\geq 1: 160)$ (Fig. 2a). Fluorescein isothiocyanate 
a Virus + serum (1:10 serum dilution)
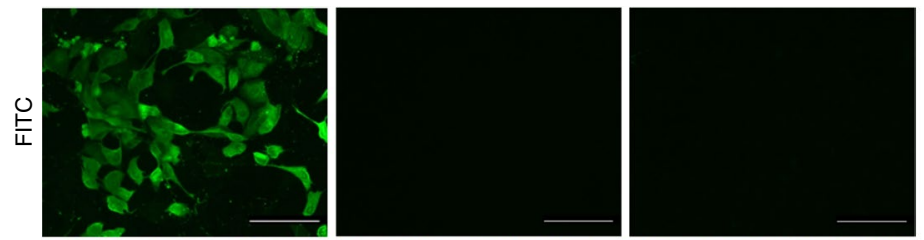

Controls
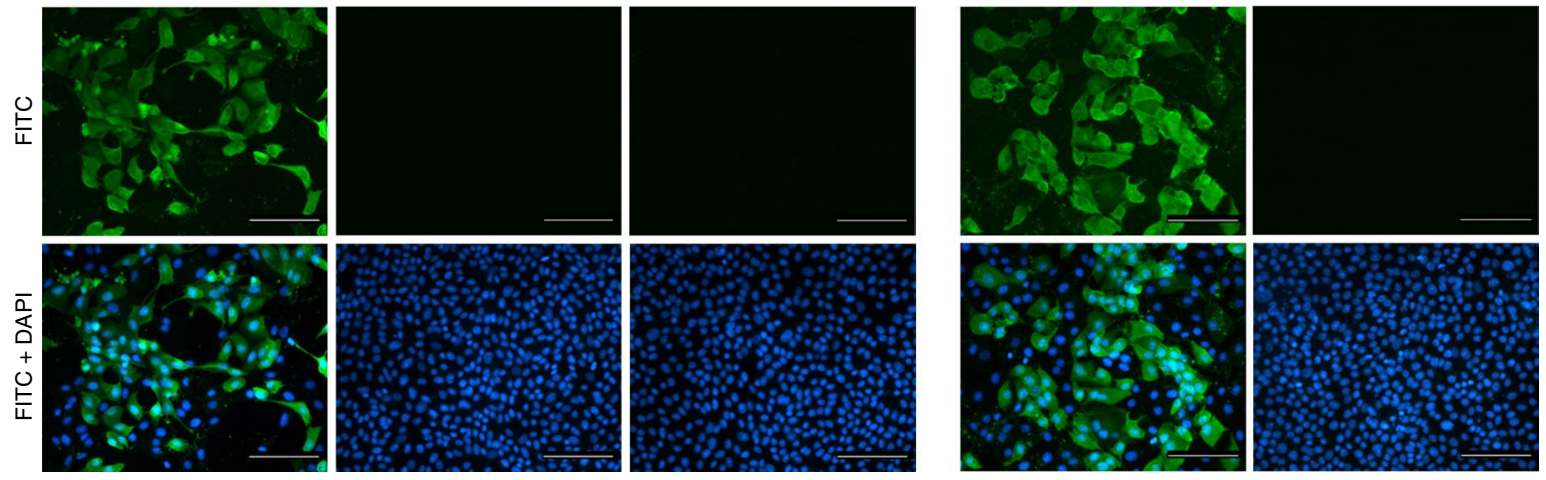

Virus + serum

(1:160 serum dilution)
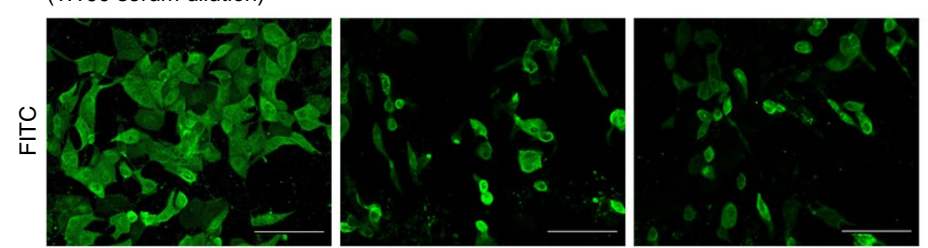

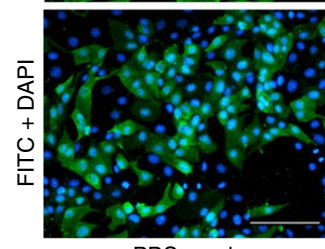

PBS-mock

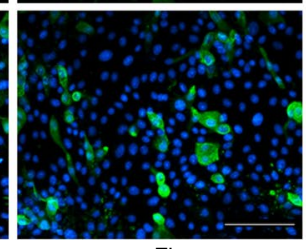

$\gamma$-Flu

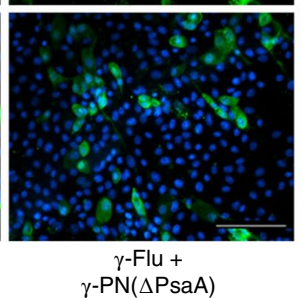

b

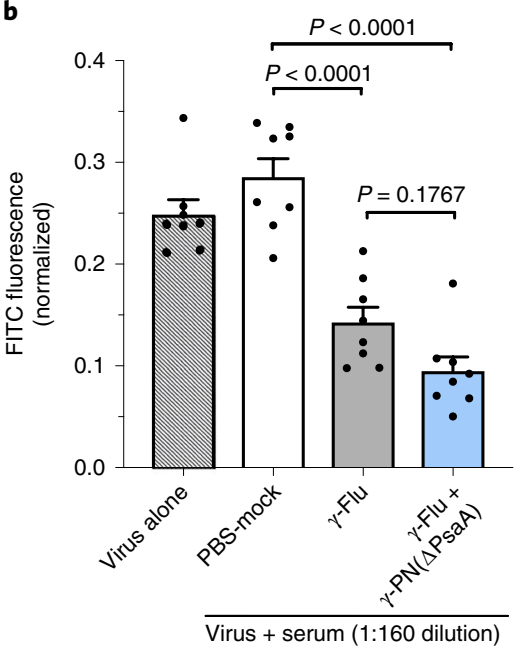

Fig. 2 | In vitro neutralization of A/PR8 by vaccine-induced antibodies. Serum was collected on day 20 post-vaccination from mice receiving $\gamma$-Flu alone, $\gamma$-Flu $+\gamma$-PN $(\Delta$ PsaA $)$ or a PBS-mock vaccine. Serum samples were pooled for each group and serially diluted, and then incubated with live A/ PR8. Virus + serum mixtures were added to MDCK cell monolayers at MOI 0.1 to assess neutralization. a, Representative images from two biologically independent experiments of infected cells; DAPI (blue) indicates cell nuclei, and FITC (green) indicates IAV. Control wells were incubated with virus alone (no serum), or allantoic fluid alone. Scale bars, $100 \mu \mathrm{m}$. b, Neutralization of IAV tested as above, using individual serum samples at 1:160 dilution. FITC fluorescence was quantified for each sample using NIS elements software, and normalized using corresponding quantified DAPI fluorescence. The data are presented as mean \pm s.e.m. ( $n=8$ biologically independent serum samples per group), and were analysed by one-way ANOVA with Tukey's multiple comparisons test. The data are representative of two biologically independent experiments.

(FITC) fluorescence (indicating IAV infection) was quantified at this dilution, and a significant reduction was detected following treatment with sera from each vaccine group when compared to sera from PBS-mock controls (Fig. 2b). Importantly, comparable neutralization was detected for both vaccine groups. We also tested whether $\gamma$-Flu $+\gamma$-PN $(\Delta$ PsaA $)$ co-administration facilitated the induction of cross-neutralizing antibodies. Serum was incubated with a fixed titre of live pdmH1N1 (drifted) before infection of monolayers. Interestingly, the data show that neither immune sera nor control sera had any neutralizing activity against pdmH1N1 (Supplementary Fig. 1), indicating that the reduction in clinical symptoms in co-vaccinated mice after pdmH1N1 challenge was probably due to augmented T-cell immunity, rather than antibody responses.

Co-vaccination has minimal impact on circulating T-cell responses. To investigate alterations in IAV-specific cell-mediated immunity, the OT-I system was utilized. OT-I cells were adoptively transferred to $\mathrm{C} 57 \mathrm{BL} / 6$ mice, followed by vaccination with irradiated A/PR8-OVA ( $\gamma$-Flu-OVA) alone or $\gamma$-Flu-OVA + $\gamma$-PN( $\Delta$ PsaA). Peripheral blood was collected on days 7, 14 and 21 post-vaccination, as were the spleen and mediastinal lymph node $(\mathrm{mLN})$ on day 21 . Significantly enhanced frequencies of activated OT-I cells $\left(\mathrm{CD}^{+} \mathrm{CD} 45.1^{+} \mathrm{CD} 44^{\mathrm{hi}}\right)$ were detected in all tissues for $\gamma$-Flu-OVA and co-vaccinated mice compared to PBS-mock controls (Fig. $3 \mathrm{a}-\mathrm{c}$ ), indicating robust induction of systemic IAV-specific responses after a single non-adjuvanted intranasal vaccination. OT-I responses in the mLN and peripheral blood were comparable between vaccine groups, although a slight decrease in the frequency of OT-I cells in the blood of co-vaccinated mice was detected on day 7. This did not reach statistical significance, and frequencies were comparable by day 14 . Interestingly, co-administration of $\gamma$-Flu-OVA with $\gamma$-PN $(\Delta$ PsaA $)$ did significantly reduce the population of activated OT-I cells in the spleen (Fig. $3 \mathrm{~b}$ ).

Exposure to viral antigen typically induces three subsets of $\mathrm{CD}^{+} \mathrm{T}$ cells with distinct phenotypic, homeostatic and migratory properties, as characterized by Gerlach et al. ${ }^{16}$. Despite the reduced OT-I population in the spleen of co-vaccinated mice, the frequencies of central memory $\left(\mathrm{T}_{\mathrm{CM}}\right)$, peripheral memory $\left(\mathrm{T}_{\mathrm{PM}}\right)$ and effector memory $\left(\mathrm{T}_{\mathrm{EM}}\right)$ among OT-I cells were comparable between vaccine groups (Fig. 3e). Similar profiles for these subsets were 


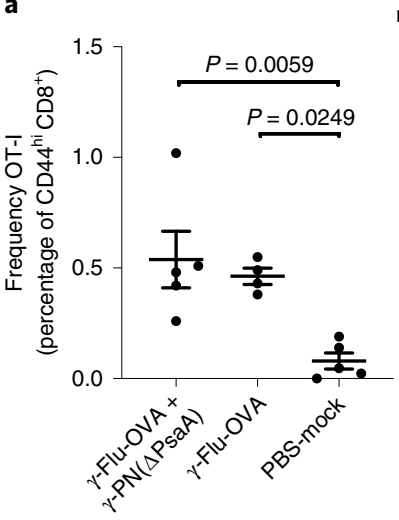

$\mathrm{mLN}$ d21

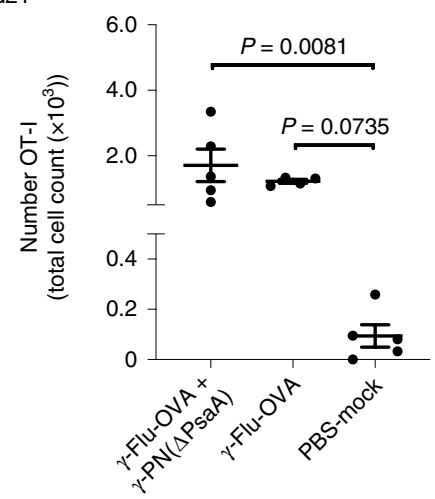

d

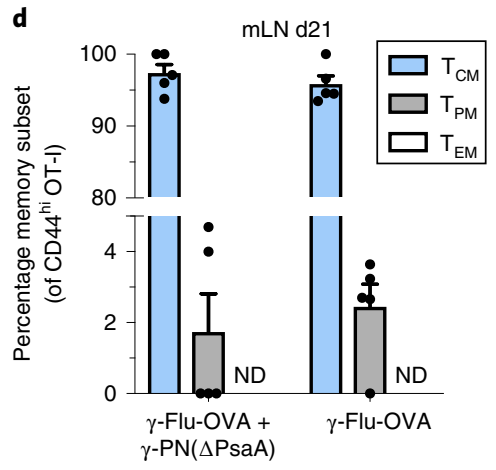

b

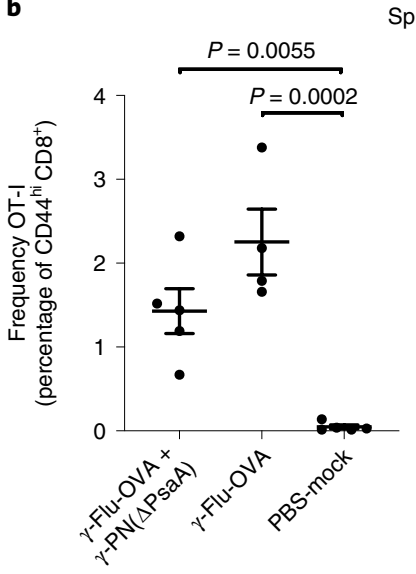

Spleen d21

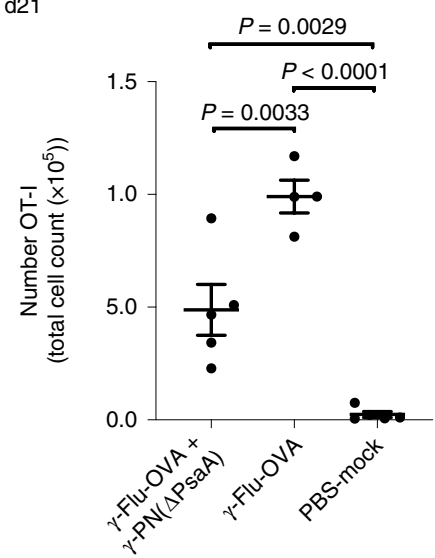

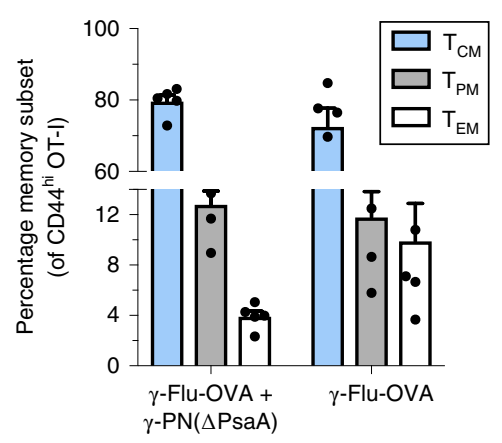

c

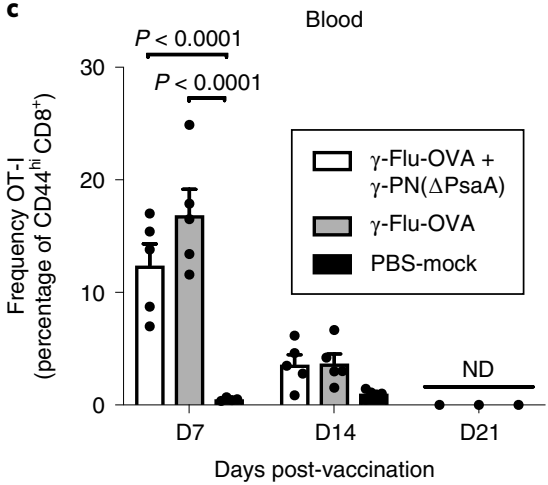

$\mathbf{f}$

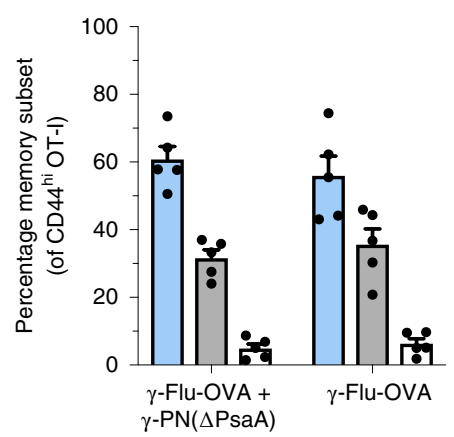

Blood d7

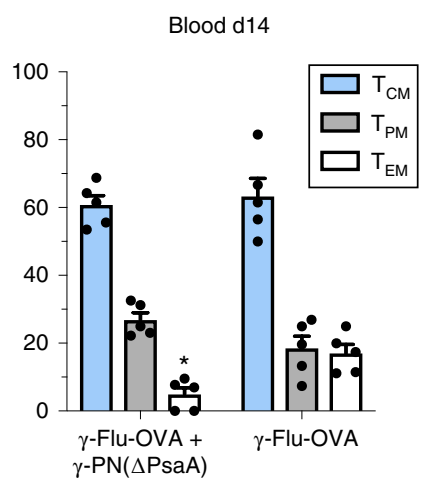

Fig. 3 | IAV-specific T-cell populations in peripheral blood and secondary lymphoid organs. OT-I cells were transferred intravenously to wild-type C57BL/6 mice. After $24 \mathrm{~h}$, mice were vaccinated i.n. with $\gamma$-Flu-OVA or co-vaccinated with $\gamma$-Flu-OVA $+\gamma$-PN $(\Delta \mathrm{PsaA})$. a,b, mLN (a) and spleen (b) were collected on day 21 post-vaccination, and activated OT-I cells (CD44hi CD8 ${ }^{+}$CD $45.1^{+}$) were quantified by flow cytometry. The data are presented as frequency and total cell counts \pm s.e.m. ( $n=5$ mice per group), and were analysed by one-way ANOVA with Tukey's multiple comparisons test. c, Blood was collected by submandibular bleed on day 7, 14 and 21 post-vaccination, and activated OT-I cells were quantified by flow cytometry. The data are presented as mean frequency \pm s.e.m. ( $n=5$ mice per group), and were analysed by two-way ANOVA with Tukey's multiple comparisons test. $\mathbf{d}-\mathbf{f}$, Singlecell suspensions from $\mathrm{mLN}(\mathbf{d})$, spleen $(\mathbf{e})$ and blood (f) were analysed for proportions of circulating memory cell subsets $\mathrm{T}_{\mathrm{CM}}\left(\mathrm{CD} 27^{+} \mathrm{CX} 3 \mathrm{CR} 1^{-}\right), \mathrm{T}_{\mathrm{PM}}$ $\left(\mathrm{CD} 27^{+} \mathrm{CX} 3 \mathrm{CR} 1^{+}\right)$and $\mathrm{T}_{\mathrm{EM}}\left(\mathrm{CD} 27^{-} \mathrm{CX} 3 \mathrm{CR} 1^{+}\right)$. The data are presented as frequency \pm s.e.m. $(n=5$ mice per group), and were analysed by two-way ANOVA with Tukey's multiple comparisons test ( ${ }^{\star} P<0.05$, when comparing the same cell subset between vaccine groups). ND, not detected.

also observed in the mLN (Fig. 3d) and peripheral blood (Fig. 3f), although a small but significant decrease in $\mathrm{T}_{\mathrm{EM}}$ cell frequency was detected in the blood of co-vaccinated mice at day 14.

Co-vaccination enhances the IAV-specific $\mathrm{CD8}^{+} \mathrm{T}$-cell response in the lungs. As circulating $\mathrm{T}$ cells in the blood and secondary lymphoid organs were not overly affected by co-vaccination, the elevated protection against IAV challenge was potentially due to lung-specific immunity. To address this, OT-I cells were transferred to $\mathrm{C} 57 \mathrm{BL} / 6$ mice before vaccination, and lungs were collected for flow cytometry on day 21 . In this tissue, a significant increase in activated OT-I cells was detected for co-vaccinated mice compared to those receiving $\gamma$-Flu-OVA alone (Fig. 4a). Lung suspensions were also stimulated ex vivo with the OVA peptide SIINFEKL to assess functionality. As shown in Fig. 4b, cells from $\gamma$-Flu-OVAvaccinated and co-vaccinated mice were equivalent in their ability 
to produce inflammatory cytokines following stimulation with cognate antigen. The co-vaccinated group tended to have slightly lower frequencies of cytokine-positive cells, but no statistically significant differences were detected. Furthermore, the mean fluorescence intensity for each cytokine was comparable between vaccine groups (Fig. 4c). Thus, activated T cells from $\gamma$-Flu-OVA-vaccinated and co-vaccinated mice appeared to be functionally equivalent, with coadministration inducing a larger bulk population of IAV-specific cells at the site of pathogen re-encounter.

In addition to the memory $\mathrm{CD} 8^{+} \mathrm{T}$-cell populations mentioned above, previous studies have identified a highly specialized subset of tissue-resident memory cells $\left(\mathrm{T}_{\mathrm{RM}}\right)$, which are retained for extended periods of time in non-lymphoid tissues including the skin, gut and lung ${ }^{17-19}$. Given the enhanced number of OT-I cells in the lungs of co-vaccinated mice, it was of interest to determine whether these cells also displayed a $\mathrm{T}_{\mathrm{RM}}$ phenotype. Both CD45.1+ OT-I cells and endogenous $\mathrm{CD} 45.1^{-} \mathrm{CD} 8^{+} \mathrm{T}$ cells were classified on the basis of the expression of the established $\mathrm{T}_{\mathrm{RM}}$ markers CD69 and $\mathrm{CD}_{103^{20}}$. Gating on these key markers is shown by representative plots in Fig. 4d. Remarkably, quantification of OT-I-derived $\mathrm{T}_{\mathrm{RM}}$ demonstrated a significant enhancement in total cell number for co-vaccinated mice compared to mice receiving $\gamma$-Flu-OVA alone (Fig. 4e). The frequency of OT-I $\mathrm{T}_{\mathrm{RM}}$ was also elevated, suggesting that, on a single-cell basis, $C D 8^{+} \mathrm{T}$ cells were more readily converted to $\mathrm{T}_{\mathrm{RM}}$ in the lung microenvironment of co-vaccinated animals.

Enhanced $\mathrm{T}_{\mathrm{RM}}$ populations were also observed for endogenous $\mathrm{CD}^{+}$and $\mathrm{CD}^{+} \mathrm{T}$ cells. In both instances, $\mathrm{T}_{\mathrm{RM}}$ populations were significantly larger in co-vaccinated mice compared to PBS-mock controls (Fig. 4f). Importantly, there was an obvious trend for covaccinated mice to have more $\mathrm{T}_{\mathrm{RM}}$ compared to mice receiving $\gamma$-Flu-OVA alone. As endogenous cells were analysed here, antigen specificity was not determined. Thus, administration of both viral and bacterial vaccines is likely to have induced multiple $T_{R M}$ populations, leading to a larger bulk number overall.

Analysis of the lung micro-environment immediately after vaccination with $\gamma$-Flu or $\gamma$-Flu $+\gamma$ - $\mathrm{PN}(\Delta \mathrm{PsaA})$ was also performed, to assess whether alterations to the cytokine milieu could contribute to the observed $\mathrm{T}_{\mathrm{RM}}$ enhancement. Bronchoalveolar lavage (BAL) samples were obtained at days 2, 4 and 6 post-vaccination for cytokine multiplex analysis. Supplementary Fig. 2 shows a synergistic enhancement in the expression of the trafficking chemokines CXCL10 and CCL2 in co-vaccinated mice compared to those receiving either vaccine alone. Significant increases in transforming growth factor beta (TGF- $\beta$ ) and tumour-necrosis factor alpha (TNF- $\alpha$; associated with $\mathrm{T}_{\mathrm{RM}}$ differentiation) were also detected in co-vaccinated animals.

Combining IAV with $\gamma-\mathrm{PN}(\Delta \mathrm{PsaA})$ is associated with enhanced viral uptake. An alternative mechanism to explain the enhanced $\mathrm{T}$-cell responses is increased antigen uptake in co-vaccinated mice, enhancing antigen presentation and stimulation of naive $\mathrm{T}$ cells. Thus, the influence of $\gamma$-PN $(\Delta$ PsaA $)$ on uptake of $\gamma$-Flu was investigated using THP-I macrophages. Macrophages were exposed to live or irradiated samples of $\gamma$-Flu, either alone or pre-incubated with $\gamma$-PN $(\Delta$ PsaA $)$. IAV internalization by these phagocytic cells was then quantified by intracellular staining with a FITCconjugated anti-IAV nucleoprotein (NP) antibody and flow cytometry. Representative histograms (Fig. 5a) show a positive shift in IAV-specific fluorescence when macrophages were incubated with IAV-containing samples (blue line), compared to incubation with media alone (grey) or $\gamma-\mathrm{PN}(\Delta \mathrm{PsaA})$ alone. The total number of $\mathrm{NP}^{+}$ macrophages was also quantified for each antigen mixture. As illustrated in Fig. 5b, pre-incubation of both live and irradiated IAV with $\gamma-\mathrm{PN}(\Delta \mathrm{PsaA})$ significantly increased the degree of viral internalization. It is important to note that all excess antigens were removed after $3 \mathrm{~h}$, and macrophages were processed and stained immediately for flow cytometry. Hence, minimal IAV replication would have occurred to skew fluorescence levels.

We then tested the effect of $\gamma-\mathrm{PN}(\Delta \mathrm{PsaA})$ on live IAV infectivity. A fixed titre of H1N1 was mixed with increasing amounts of $\gamma$-PN $(\Delta \mathrm{PsaA})$, and mixtures were added to MDCK cell monolayers. Immunofluorescent imaging demonstrated that mixing IAV with a high concentration of $\gamma$-PN $(\Delta \mathrm{PsaA})\left(10^{7}\right.$ colony forming units (CFU) equivalent per well) was associated with more dispersed viral infection compared to incubation of monolayers with IAV alone (Fig. $5 \mathrm{~d}, \mathrm{e})$. As $\gamma$ - $\mathrm{PN}(\Delta \mathrm{PsaA})$ was present only for the initial viral inoculation stage, the effect on IAV infectivity was likely to be exerted during attachment, rather than aiding release of progeny virions. This suggested a potentially direct interaction between IAV and the whole-inactivated pneumococci. Furthermore, the increased IAV-internalization by macrophages required the two vaccine components be added to cells simultaneously. Sequential exposure to each vaccine antigen did not increase the total number of IAV-positive cells (Supplementary Fig. 3), further suggesting a role for a direct pathogen-pathogen interaction.

Inactivated IAV and pneumococcal vaccines directly associate in suspension. Flow cytometry was used to assess any interaction between the whole-inactivated vaccines. In this assay, $\gamma-\mathrm{PN}(\Delta \mathrm{PsaA})$ was incubated alone or with increasing amounts of $\gamma$-Flu, and then centrifuged using speeds that would pellet pneumococci but not free virions. Thus, the presence of IAV in the pellets would indicate a direct IAV-pneumococcal interaction, allowing co-sedimentation of the substantially smaller virions with the pneumococci. Cell pellets were treated with an anti-A/PR8 antibody followed by a FITCconjugated secondary antibody, and flow cytometry was used to quantify the percentage of pneumococci that were positive for IAVspecific fluorescence. Figure 6a shows pneumococci alone (grey histogram) treated with both antibodies had minimal background fluorescence. In contrast, pre-incubation of pneumococci with $\gamma$-Flu (blue histogram) resulted in a steady increase in the amount of FITC fluorescence detected. This phenomenon was dose-dependent, and at the highest concentration of $\gamma$-Flu tested, almost $80 \%$ of all pneumococci within suspension were IAV-bound (Fig. 6b). As an added control, IAV was incubated and centrifuged in the absence of pneumococci. While 10,000 events were readily detected in all pneumococci-containing samples, IAV alone returned $<150$ events in total in an equivalent volume (Supplementary Fig. 3 ). Thus, the low-speed centrifugation used for this assay was unable to independently pellet IAV virions to contribute to the IAV-positive events detected. Furthermore, equivalent levels of binding were observed when mixing live or irradiated IAV and pneumococci, indicating that our method of vaccine inactivation successfully maintains native pathogen-pathogen interactions.

To further assess the direct interaction between the wholeinactivated vaccines, transmission electron microscopy (TEM) was employed. Negative staining and TEM revealed both IAV virions and pneumococcal cells within pelleted samples, with multiple virions bound to the surface of individual pneumococci (Fig. 6c).

\section{Discussion}

We have previously demonstrated the enhanced immunogenicity of $\gamma$-PN when co-administered with $\gamma$-Flu ${ }^{15}$. Remarkably, the current study shows that $\gamma$-PN $(\Delta$ PsaA) similarly enhances $\gamma$-Flu-specific responses following co-administration. While $\gamma$-Flu-vaccinated and $\gamma$-Flu $+\gamma$-PN $(\Delta$ PsaA $)$ co-vaccinated animals showed comparable homotypic protection, co-vaccinated animals showed significantly elevated protection against drifted and heterosubtypic IAV (Fig. 1). Interestingly, immune sera from both vaccinated groups showed equivalent in vitro neutralization of homotypic H1N1 and no crossneutralization against drifted pdmH1N1, indicating that the differential immunity was T-cell-based. In fact, our previous studies 
a

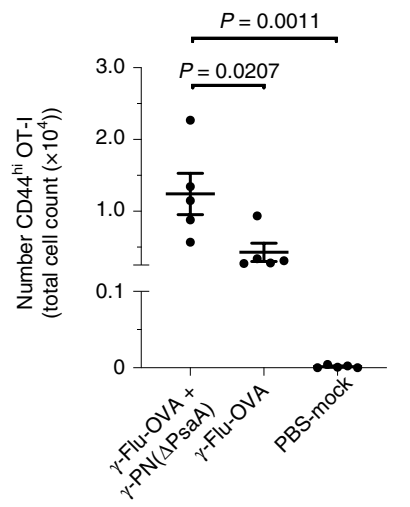

b

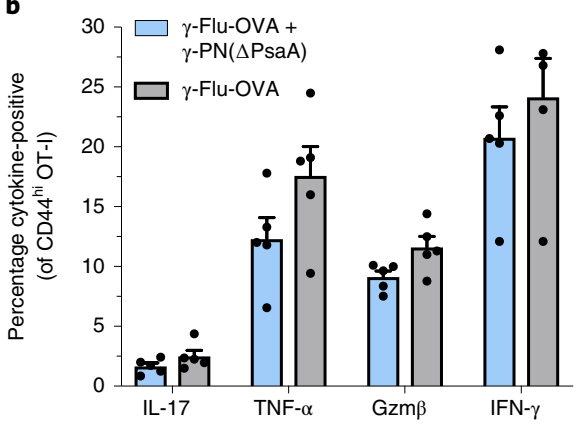

C

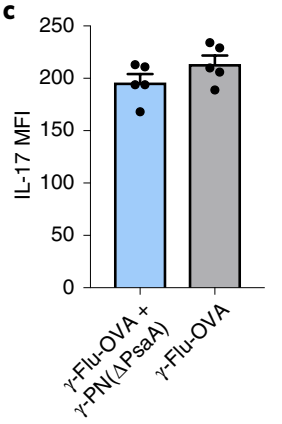

d
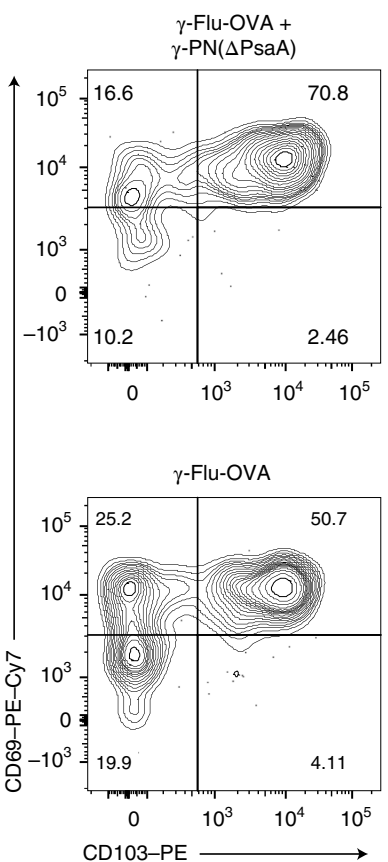
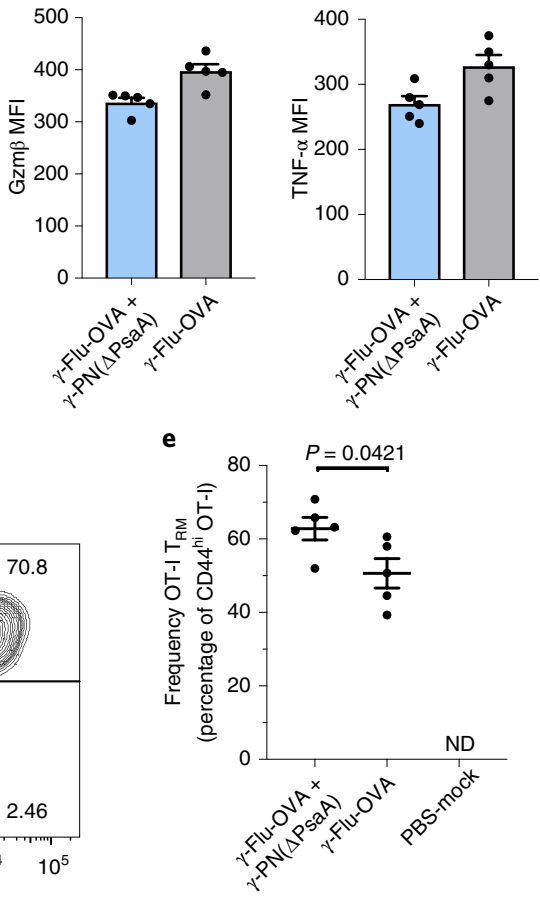

$\mathbf{f}$

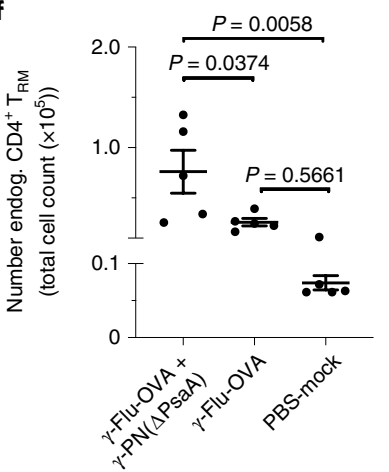

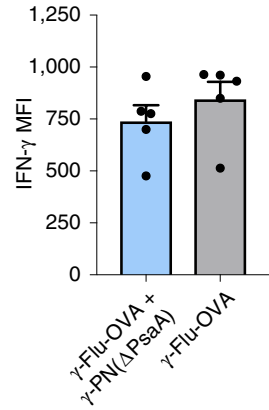
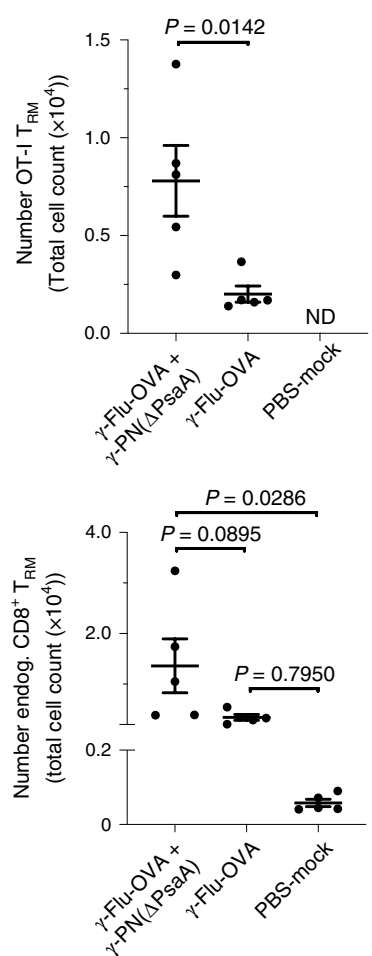

Fig. 4 | The magnitude of the IAV-specific CD8+ T-cell response in the lung is enhanced by co-vaccination. OT-I cells were transferred intravenously to wild-type C57BL/6 mice. After $24 \mathrm{~h}$, mice were vaccinated i.n. with $\gamma$-Flu-OVA or co-vaccinated with $\gamma$-Flu-OVA $+\gamma$-PN $(\Delta$ PsaA). On day 21 postvaccination, lungs were collected for analysis of OT-I cells by flow cytometry. $\mathbf{a}$, The total number of activated OT-I cells $\left(C D 44^{\text {hi }} C D 88^{+} C D 45.1^{+}\right)$, presented as mean \pm s.e.m. ( $n=5$ mice per group). The data were analysed by one-way ANOVA with Tukey's multiple comparisons test. $\mathbf{b}$, Single-cell lung suspensions were stimulated ex vivo for $4 \mathrm{~h}$ with SIINFEKL peptide before intracellular cytokine staining and flow cytometry. Data presented as mean frequency \pm s.e.m. ( $n=5$ mice per group) of cytokine-positive OT-I cells (no significance was determined between vaccine groups by two-way ANOVA with Tukey's multiple comparisons test). c, Geometric mean fluorescence intensity (MFI) \pm s.e.m. ( $n=5$ mice per group) for each individual cytokine (no significance was determined between vaccine groups by two-tailed unpaired $t$-test). $\mathbf{d}$, Activated OT-I cells (CD44 ${ }^{\text {hi }}$ CD $45.1^{+}$) and endogenous T cells $\left(C D 44^{\text {hi }} C D 45.1^{-}\right)$were analysed for $T_{R M}$ phenotype $\left(C D 69^{+} C D 103^{+}\right)$by flow cytometry. Representative plots of $T_{R M}$ gating on OT-I cells are presented. e, OT-I TRM cells in the lungs of vaccinated mice were quantified, and are presented as frequency and total cell count (mean \pm s.e.m., $n=5$ mice per group). The data were analysed by two-tailed unpaired $t$-test. f, Endogenous CD4 ${ }^{+} T_{R M}$ cells (CD11a ${ }^{\text {hi }}$ CD69 $9^{+}$) (left panel) and CD8 ${ }^{+} T_{R M}$ cells (CD69+ $C D 103^{+}$) (right panel), presented as total cell count (mean \pm s.e.m., $n=5$ mice per group), and analysed by one-way ANOVA with Tukey's multiple comparisons test. 


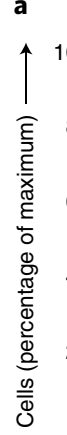
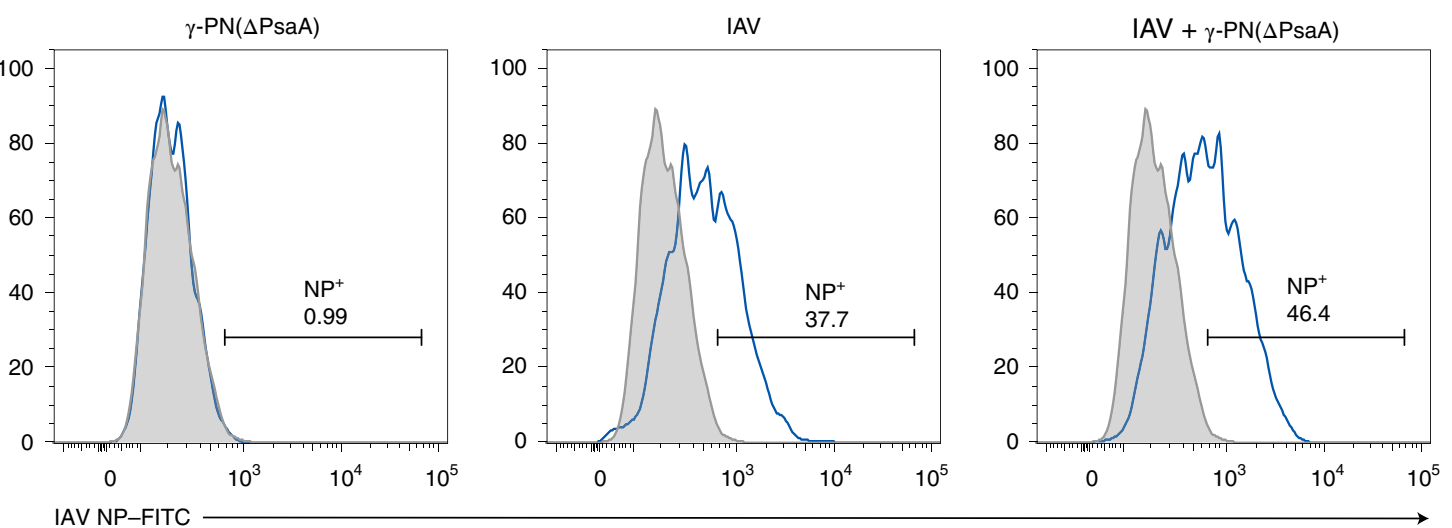

b
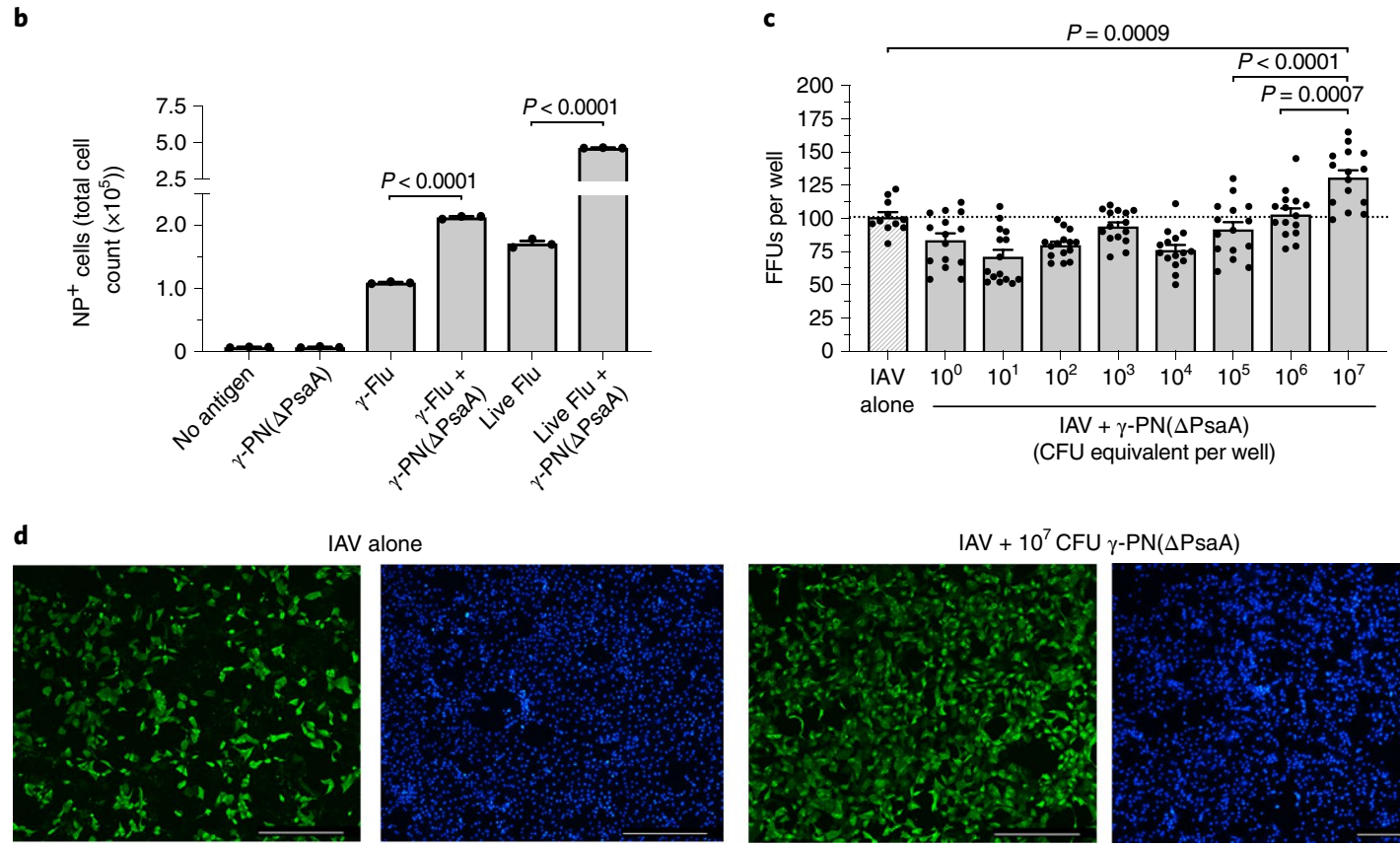

FITC

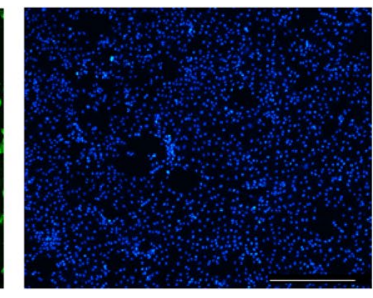

DAPI

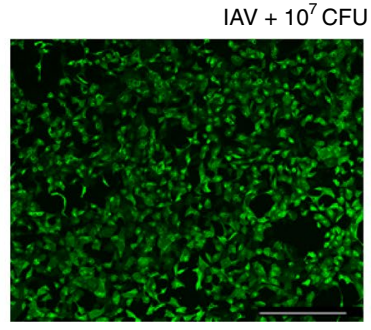

FITC

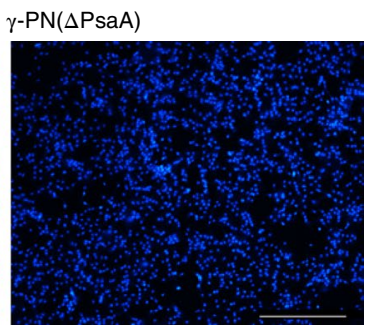

DAPI

Fig. 5 | The presence of $\boldsymbol{\gamma}$-PN( $\Delta$ PsaA) enhances the uptake of IAV by the THP-1 and MDCK cell lines. a, Differentiated THP-1 macrophage-like cells were incubated with IAV and $\gamma$-PN ( $\Delta$ PsaA) vaccines (either alone or in combination) for $3 \mathrm{~h}$. Cells were extensively washed to remove unbound antigen and then processed for analysis by flow cytometry. Representative histograms from two biologically independent experiments are shown. FITC fluorescence indicates intracellular IAV NP after incubation with medium alone (grey) or IAV $\pm \gamma$-PN $(\Delta$ PsaA) (blue). b, THP-1 macrophage-like cells were incubated with live or irradiated IAV $\pm \gamma-\mathrm{PN}(\Delta \mathrm{PsaA})$, and total $\mathrm{NP}^{+}$cells were quantified for each antigen mixture. The data are presented as mean $\pm \mathrm{s}$.e.m. $(n=3$ biologically independent samples per group), and were analysed by one-way ANOVA with Tukey's multiple comparisons test. The data are representative of two biologically independent experiments. c, Increasing concentrations of $\gamma$-PN( $\triangle P$ saA) were added to live IAV, and mixtures were added to MDCK monolayers at 100 FFUs per well of IAV. FFU per well was quantified after incubation and immunofluorescent staining. The data are presented as mean \pm s.e.m. ( $n=15$ biologically independent samples for each test group, $n=11$ for virus alone control), and were analysed by one-way ANOVA with Tukey's multiple comparisons test. The data are representative of two biologically independent experiments. d, Fluorescent images of MDCK monolayers after incubation with live IAV alone or IAV in combination with $10^{7} \mathrm{CFU}$ equivalent $\gamma$-PN $(\Delta$ PsaA). Virus was added to monolayers at MOI 0.1 in both cases. FITC (green) indicates IAV, and DAPI (blue) indicates cell nuclei. The images are representative of two biologically independent experiments. Scale bars, $500 \mu \mathrm{m}$.

have established a crucial role for $\mathrm{CD} 8^{+} \mathrm{T}$ cells rather than antibody responses in $\gamma$-Flu-mediated cross-protection ${ }^{13}$.

The data presented here demonstrate that co-vaccination enhances IAV-specific T-cell immunity specifically at the site of pathogen re-encounter. OT-I cells isolated from the lung of $\gamma$-FluOVA-vaccinated and co-vaccinated mice showed equivalent functionality; however, a larger bulk population of activated cells was present in co-vaccinated animals (Fig. 4). As the larger OT-I population in the lung was paired with a decreased population in the spleen (Fig. 3b), it is unlikely that total activation of IAV-specific cells was greater in either vaccine group. Rather, subsequent recruitment to the lung appears to have been improved by co-vaccination. In fact, the trafficking chemokines CXCL10 and CCL2 were synergistically enhanced in the BAL of co-vaccinated mice compared to those receiving $\gamma$-Flu alone (Supplementary Fig. 2). CCL2 promotes tissue infiltration of inflammatory monocytes/macrophages $^{21}$, while CXCL10 and its receptor CXCR3 have been implicated in recruitment of $\mathrm{T}$ cells to the lung following respiratory infection $^{22,23}$. A higher frequency of lung OT-I cells also showed a $\mathrm{T}_{\mathrm{RM}}$ phenotype in co-vaccinated animals (Fig. 4e). Numerous 
a
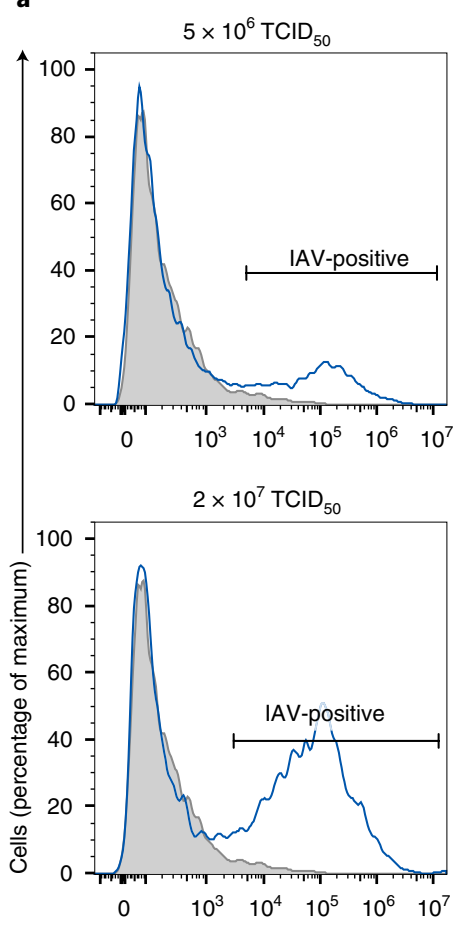

IAV-FITC
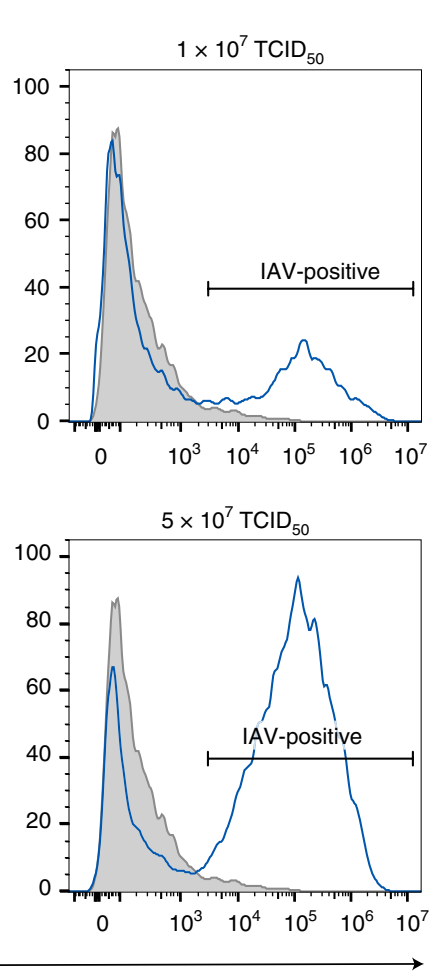

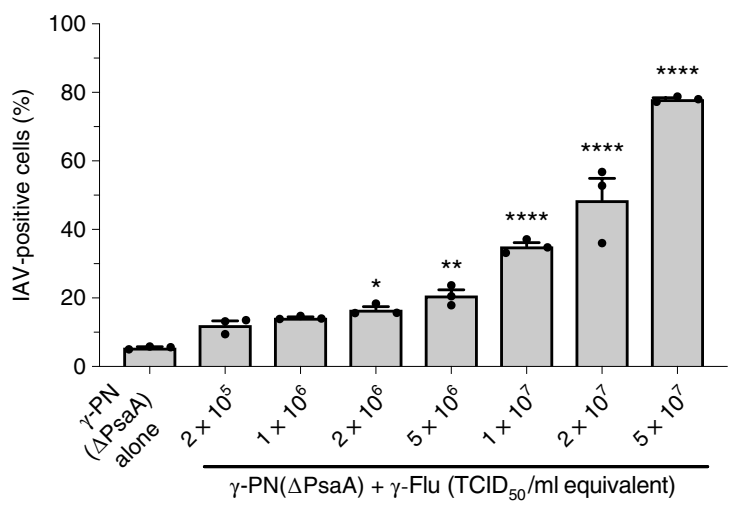

c

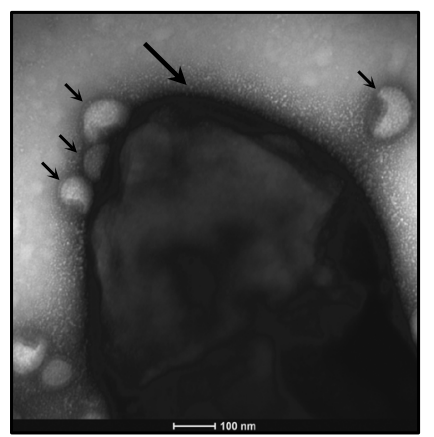

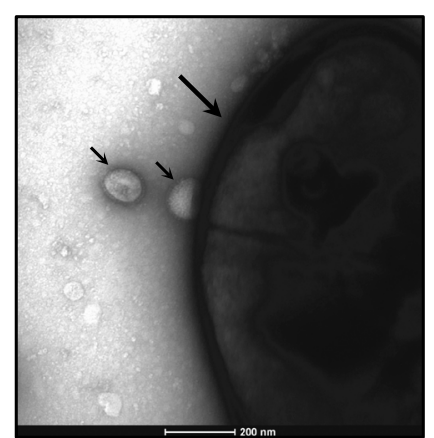

Fig. 6 | Direct association of $\gamma$-Flu and $\gamma$-PN $(\Delta$ PsaA) whole-inactivated vaccines. $\mathbf{a}, \gamma$-PN $(\Delta$ PsaA) was mixed with increasing amounts of $\gamma$-Flu, and incubated statically for $30 \mathrm{~min}$ at $37^{\circ} \mathrm{C}$. Unbound virions were removed by pelleting and extensive washing of pneumococci. Pneumococci were then stained with an IAV-specific FITC-conjugated antibody, and single pneumococcal cells were analysed by flow cytometry. Representative plots of FITC fluorescence (indicating IAV) are presented for $\gamma-\mathrm{PN}(\triangle \mathrm{PsaA})$ alone (grey) or $\gamma-\mathrm{PN}(\triangle \mathrm{PsaA})$ incubated with increasing concentrations $\left(T_{C I D_{50}}\right.$ equivalent $\mathrm{ml}^{-1}$ ) of $\gamma$-Flu (blue). The histograms are representative of two biologically independent experiments. $\mathbf{b}$, The percentage of IAV-positive pneumococci was quantified for each $\gamma$-Flu concentration tested. The data are presented as mean \pm s.e.m. ( $n=3$ biologically independent samples per group), and were analysed by one-way ANOVA with Tukey's multiple comparisons test $\left({ }^{\star} P<0.05,{ }^{\star \star} P<0.01,{ }^{\star \star \star \star} P<0.0001\right.$, compared to $\gamma-P N(\Delta P s a A)$ alone). The data are compiled from two independent experiments. $\mathbf{c}, \gamma$-Flu-OVA and $\gamma$-PN $(\Delta \mathrm{PsaA})$ vaccines were mixed, and then washed and negatively stained for imaging by TEM. Representative images from two biologically independent experiments are presented. Small arrows indicate individual IAV virions and large arrows indicate pneumococci.

studies have demonstrated that lung-resident $\mathrm{T}_{\mathrm{RM}}$ cells are crucial for protection against respiratory viral infection, including $\mathrm{IAV}^{24,25}$. Conversion to $\mathrm{T}_{\mathrm{RM}}$ requires a variety of tissue-derived factors, including TGF- $\beta$, interleukin-15 (IL-15), TNF and IL-33 ${ }^{19,26-29}$. Intranasal S. pneumoniae infection can induce TNF in the lung ${ }^{30}$, and low pneumococcal carriage density causes elevation of nasopharyngeal TGF- $\beta 1^{31}$. Supplementary Fig. 2 shows enhancement of both these cytokines in the BAL of co-vaccinated animals compared to those receiving $\gamma$-Flu alone, and data indicate that these elevated levels are predominately due to the $\gamma-\mathrm{PN}(\Delta \mathrm{PsaA})$ vaccine component. Thus, co-vaccination enhances cytokines crucial for both $\mathrm{CD}^{+} \mathrm{T}$-cell trafficking to the lung and subsequent conversion to a $\mathrm{T}_{\mathrm{RM}}$ phenotype.

The longevity of vaccine-induced $\mathrm{T}_{\mathrm{RM}}$ detected here is also of interest, especially considering reduced $\mathrm{T}_{\mathrm{EM}}$ populations in the blood and spleen of co-vaccinated mice (Fig. 3e,f). A study by Slutter et al. suggests that circulating $\mathrm{T}_{\mathrm{EM}}$ are the precursors to lung $\mathrm{T}_{\mathrm{RM}}{ }^{32}$. The reduction in $\mathrm{T}_{\mathrm{EM}}$ may therefore be due to rapid recruitment from the circulation to the lung tissue. However, if this is not the case and the $\mathrm{T}_{\mathrm{EM}}$ pool is consistently smaller in co-vaccinated animals, the overall longevity of the $\mathrm{T}_{\mathrm{RM}}$ population may be affected. We therefore intend to assess the kinetics of IAV-specific $T_{R M}$ in $\gamma$-Flu-vaccinated and co-vaccinated mice at later time points in a follow-up study.

Interactions with other lymphocytes could also contribute to the enhanced $\mathrm{CD}^{+} \mathrm{T}$-cell responses observed. Previous studies by our group have shown increased interferon- $\gamma^{+}\left(\mathrm{IFN}-\gamma^{+}\right) \mathrm{CD} 4^{+}$ T-cell populations in the lungs of $\gamma$-Flu $+\gamma$-PN co-vaccinated mice compared to controls receiving $\gamma$-Flu alone ${ }^{15}$. $\mathrm{CD}^{+} \mathrm{T}$ cells and IFN- $\gamma$ can facilitate tissue entry of $\mathrm{CD}^{+} \mathrm{T}$ cells $\mathrm{s}^{33,34}$, and are required for the expression of CXCR3 and CD103 by CD8 ${ }^{+} \mathrm{T}$ cells ${ }^{33}$. Here, increased $\mathrm{CD}^{+}$resident memory $\mathrm{T}$-cell populations were detected in the lungs of co-vaccinated mice (Fig. 4f). These cells could mediate $\mathrm{CD}^{+} \mathrm{T}$-cell infiltration, or could directly confer viral protection via perforin-dependent cytotoxicity ${ }^{35}$. CD $4^{+}$effectors have been shown to kill IAV-peptide-coated targets in cytolytic assays, and confer protection in mice against IAV challenge ${ }^{36}$. CD $4^{+} \mathrm{T}$ cells isolated from IAV-infected human volunteers also showed cytotoxicity against IAV-peptide-pulsed targets, and the frequency of circulating $\mathrm{CD}_{4}^{+} \mathrm{T}$ cells correlated with reduced illness duration and viral shedding following challenge ${ }^{37}$. Interaction with antigenpresenting cells may additionally contribute to augmented immunity in co-vaccinated mice. Our data show enhanced viral uptake by macrophages in vitro when live IAV and $\gamma$-Flu virions were mixed with $\gamma-\mathrm{PN}(\Delta \mathrm{PsaA})$ (Fig. 5a,b). Although dendritic cells are the predominant antigen-presenting cell participating in naive T-cell stimulation, peptide-pulsed macrophages can induce $\mathrm{CD}^{+}$ T-cell proliferation and differentiation ${ }^{38}$. Macrophages were also found to undergo necrosis-like death in response to ultravioletinactivated A/PR8 and heat-killed S. pneumoniae; this was associated with activation and relocation of $\mathrm{CD} 11 \mathrm{~b}^{+}$dendritic cells $\mathrm{s}^{39}$. The increased antigen uptake observed here could therefore heighten 
T-cell activation, and may promote dendritic cell trafficking for superior antigen presentation. Our data also demonstrate enhanced viral infection of epithelial-like cells when pre-incubating IAV with high $\gamma$-PN $(\Delta$ PsaA) concentrations (Fig. 5c,d). Co-vaccination with $\gamma-\mathrm{PN}(\Delta \mathrm{PsaA})$ may similarly increase internalization of $\gamma$-Flu virions by epithelial cells in vivo, as $\gamma$-Flu is able to undergo membrane fusion due to intact virion morphology and functional surface proteins. This would amplify the amount of intracellular IAV antigen available for presentation on major histocompatibility complex I and subsequent induction of cytotoxic T-cell responses.

Astoundingly, direct binding between the functionally intact, but inactivated, IAV and pneumococcal vaccines may underlie the increased vaccine immunogenicity. Simultaneous exposure to $\gamma$-Flu and $\gamma-\mathrm{PN}(\Delta \mathrm{PsaA})$ was required for enhanced uptake by macrophages, whereas adding the vaccine antigens sequentially had no significant effect (Supplementary Fig. 3). When considering phagocytic uptake, recognition and internalization of the entire IAV-pneumococcal complex is likely. This would induce a dramatically altered cytokine profile compared to $\gamma$-Flu alone due to the presence of additional bacterial pathogen-associated molecular patterns. For example, IAV-infected human monocyte-derived macrophages were shown to upregulate production of multiple inflammatory mediators after subsequent $S$. pneumoniae exposure, particularly CXCL $10^{40}$.

While synergistic interactions between S. pneumoniae and other respiratory viruses (respiratory syncytial virus, parainfluenza viruses and human metapneumovirus) have previously been reported $^{41-43}$, this report demonstrates a direct IAV-pneumococcal interaction. Interestingly, binding between IAV and other streptococci has been observed previously, with the interaction being dependent on streptococcal capsular polysaccharide (CPS). Okamoto et al. reported that binding between IAV and Streptococcus pyogenes was substantially reduced when the bacterial capsule was removed $^{44}$. A sialic acid moiety in Streptococcus suis CPS similarly mediates binding to swine influenza viruses ${ }^{45}$, and a capsular sialic acid-dependent interaction with IAV was shown for Streptococcus agalactiae $^{46}$. Strikingly, these interactions are in direct contrast to data presented here. Due to use of a non-encapsulated pneumococcal vaccine strain, our data demonstrate a high degree of binding between IAV and $S$. pneumoniae in the complete absence of pneumococcal CPS. Pneumococcal surface proteins may therefore mediate the binding observed. In addition, kinetics data in Supplementary Fig. 3 show a small degree of binding immediately after mixing the vaccine components, suggesting that hydrophobic interactions could be contributing.

Overall, this study demonstrates the immense value of utilizing both known and novel aspects of the synergism between IAV and S. pneumoniae, and exploiting them for superior vaccine-induced responses. The dual enhancement of responses demonstrated here and in our previous study ${ }^{15}$, paired with the conferral of broadspectrum protection, makes this co-vaccination strategy ideal for limiting mortality during future influenza pandemics and seasonal epidemics. An increased understanding of the mechanisms permitting pathogen-pathogen interactions may also shed light on disease progression during co-infection, and assist in the development of new therapeutics to mitigate the severity of symptoms.

\section{Methods}

Bacterial and viral vaccine stocks. Influenza A viruses (A/Puerto Rico/8/34 (H1N1) (A/PR8)) and A/PR8-OVA were grown in the allantoic cavity of 10-day-old embryonated chicken eggs. A/PR8-OVA is engineered to express the ovalbumin peptide SIINFEKL (the original stock was a gift from S. Turner, Monash University). Eggs were injected with $10^{3}$ half-maximum tissue-culture infectious dose $\left(\mathrm{TCID}_{50}\right)$ of virus, incubated for $48 \mathrm{~h}$ at $37^{\circ} \mathrm{C}$, and chilled at $4{ }^{\circ} \mathrm{C}$ overnight. Allantoic fluid was collected, pooled and stored at $-80^{\circ} \mathrm{C}$. Virus stocks were then concentrated using chicken red blood cells (cRBCs), as previously described ${ }^{4}$. Briefly, allantoic fluid was incubated with cRBCs for 45 min at $4{ }^{\circ} \mathrm{C}$ to allow binding of viral haemagglutinin to erythrocytes, then centrifuged for $10 \mathrm{~min}$ at $3,200 \mathrm{~g}\left(\right.$ at $\left.4{ }^{\circ} \mathrm{C}\right)$, and allantoic supernatant was removed. Pellets were resuspended in $0.85 \%$ saline, incubated for $1.5 \mathrm{~h}$ at $37^{\circ} \mathrm{C}$ to release virus from cRBCs, and then centrifuged to separate erythrocytes from virus-containing supernatant. Concentrated stocks were titrated in MDCK cells using $\operatorname{TCID}_{50}$ assay ${ }^{48}$ and virus titres were estimated to be $2 \times 10^{8} \mathrm{TCID}_{50} \mathrm{ml}^{-1}$ for A/PR8, and $6 \times 10^{6} \mathrm{TCID}_{50} \mathrm{ml}^{-1}$ for A/PR8-OVA.

Streptococcus pneumoniae strains were statically grown in Todd-Hewitt broth supplemented with $0.5 \%$ yeast extract (THY) at $37^{\circ} \mathrm{C}$ in $5 \% \mathrm{CO}_{2}$ unless otherwise stated. The S. pneumoniae vaccine strain used in this study (Rx1) is a capsule-deficient derivative of D39 (serotype 2). The isogenic mutant derivative $\operatorname{Rx} 1(\Delta \mathrm{Lyt} A, \mathrm{PdT})$ was generated as previously described ${ }^{49}$. Additional genetic manipulation was performed on strain $\mathrm{Rx} 1(\Delta$ LytA, $\mathrm{PdT})$ to delete the pneumococcal surface antigen A $(p s a A)$ gene in-frame, in a similar manner as previously described ${ }^{50}$. All PCR primers used are listed in Supplementary Table 1. First, a tagged $p s a A$ deletion mutant was generated by transformation of $\operatorname{Rx} 1(\Delta \mathrm{LytA}, \mathrm{PdT})$ with a cassette comprised of an erythromycin resistance gene $\left(\right.$ Ery $\left.^{\mathrm{R}}\right)$ fused to $p s a A 5^{\prime}$ and $3^{\prime}$ flanking regions. The 5' flanking region of $p s a A$ was obtained using the primers PsaAuF and PsaAuR-J214, while the 3'flanking region was obtained using the primers LM8-J215 and PsaAdR. The Ery ${ }^{\mathrm{R}}$ gene was amplified using the primers J214 and J215. The cassette was assembled by overlap extension PCR with the primers PsaAuF and PsaAdR, and used to transform $\operatorname{Rx} 1(\Delta$ LytA, PdT). All transformation steps and subsequent growth steps with the resultant $\operatorname{Rx} 1\left(\Delta\right.$ LytA, PdT, $\Delta$ PsaA::Ery $\left.{ }^{\mathrm{R}}\right)$ strain used THY supplemented with $400 \mu \mathrm{M} \mathrm{MnCl}_{2}$ to overcome the growth defect of PsaA-null mutants. A PCR product that fused the $p s a A 5^{\prime}$ and $3^{\prime}$ flanking regions was then generated via amplification of the 5' psaA flanking region with the primers PsaAuF and PsaAuRtuF, and the $3^{\prime}$ region with the primers LM8tdR and PsaAdR. These flanks were joined via overlap extension PCR with the primers PsaAuF and PsaAdR, and the resulting PCR product was used to replace the $\mathrm{Ery}^{\mathrm{R}}$ cassette in $\mathrm{Rxl}(\Delta \mathrm{Lyt} \mathrm{A}$, PdT, $\Delta$ PsaA::Ery ${ }^{R}$ ), thus deleting $p s a A$ in-frame. Successful transformants were screened for loss of erythromycin resistance by replica plating onto blood agar plates containing $\mathrm{MnCl}_{2}$, or $\mathrm{MnCl}_{2}+$ erythromycin. The $p s a A$ flanking regions of putative mutants were PCR-amplified, and in-frame deletions were confirmed by sequencing. The final $\operatorname{Rx} 1(\Delta \mathrm{Lyt} A, \mathrm{PdT}, \Delta \mathrm{PsaA})$ strain was additionally validated using PCR, Sanger sequencing and western blot.

$\operatorname{Rxl}\left(\Delta\right.$ LytA, PdT, $\Delta$ PsaA) was then grown in THY $+400 \mu \mathrm{M} \mathrm{MnCl}_{2}$ at $37^{\circ} \mathrm{C}+5 \% \mathrm{CO}_{2}$ to $\mathrm{OD}_{600 \mathrm{~nm}}=0.65$. Cells were pelleted by centrifugation at $12,000 \mathrm{~g}$ for $10 \mathrm{~min}$ at $4^{\circ} \mathrm{C}$, then washed three times in PBS and resuspended in PBS $+13.33 \%$ glycerol at a density of $\sim 10^{10} \mathrm{CFU} \mathrm{ml}^{-1}$ in $200 \mu \mathrm{l}$ aliquots.

Generation of whole-inactivated vaccines. Concentrated A/PR8, A/PR8-OVA and $\operatorname{Rx} 1(\Delta$ LytA, PdT, $\Delta \mathrm{PsaA})$ stocks were inactivated by exposure to $50 \mathrm{kGy}$, $25 \mathrm{kGy}$ and $16 \mathrm{kGy}$, respectively, of $\gamma$-radiation from the ${ }^{60} \mathrm{Co}$ irradiation facility at the Australian Nuclear Science and Technology Organisation. All samples were kept frozen on dry-ice during irradiation and transportation. Sterility of irradiated A/PR8 $(\gamma$-Flu) and irradiated A/PR8-OVA ( $\gamma$-Flu-OVA) was confirmed by passages in embryonated chicken eggs as recommended by WHO (World Health Organization) for flu vaccine manufacturing ${ }^{51}$. In brief, 10 -day-old embryonated eggs were inoculated with $100 \mu \mathrm{l}$ of inactivated virus preparation and incubated for 2 days at $37^{\circ} \mathrm{C}$. The allantoic fluid of individual eggs was then collected and used to infect new 10-day-old embryonated eggs. This process was repeated three times and lack of detectable haemagglutination in allantoic fluid from all three passages indicated complete loss of viral infectivity. To determine haemagglutination, collected allantoic fluid was serially diluted in PBS using a 96-well roundbottom plate and $0.8 \% \mathrm{cRBCs}$ in PBS were added. Plates were incubated at $4{ }^{\circ} \mathrm{C}$ and haemagglutination patterns were analysed $24 \mathrm{~h}$ later. Sterility of irradiated $\operatorname{Rx} 1(\Delta$ LytA, PdT, $\Delta$ PsaA $)(\gamma$-PN $(\Delta$ PsaA $))$ was determined by lack of detectable $\mathrm{CFU}$ after plating of neat samples on blood agar plates.

Mice and vaccinations. For challenge experiments, 6-7-week-old female wildtype BALB/c mice were suplied by Laboratory Animal Services at the University of Adelaide. Mice were first anaesthetized intraperitoneally with $10 \mu \mathrm{lg}^{-1}$ body weight ketamine anaesthetic ( $1 \%$ xylazine, $10 \%$ ketamine in sterile $\left.\mathrm{H}_{2} \mathrm{O}\right)$. Anaesthetized mice were then vaccinated intranasally (i.n.) with either $\gamma$-Flu alone $\left(6.4 \times 10^{6}\right.$ $\mathrm{TCID}_{50}$ equivalent per mouse in $\left.32 \mu \mathrm{l}\right)$ or $\gamma$-Flu $+\gamma$-PN $(\Delta \mathrm{PsaA})\left(6.4 \times 10^{6} \mathrm{TCID}_{50}\right.$ equivalent $+10^{8} \mathrm{CFU}$ equivalent $\gamma-\mathrm{PN}(\Delta \mathrm{PsaA})$ in $\left.32 \mu \mathrm{l}\right)$. Control animals received $\gamma$-PN $\left(\Delta\right.$ PsaA) $\left(10^{8} \mathrm{CFU}\right.$ equivalent in $\left.32 \mu \mathrm{l}\right)$, or plain PBS (mock-vaccine). Where necessary, inactivated vaccine components were mixed and incubated on ice for $\sim 15$ min before immunization. Serum was collected from all mice via submandibular bleeding on day 20 post-vaccination. On day 21 post-vaccination, animals were anaesthetized, and challenged i.n. with either A/PR8 (homotypic H1N1, $1.6 \times 10^{2}$ $\mathrm{TCID}_{50}$ per mouse), A/California/07/09 ((A/Cal), drifted pdmH1N1, $1.3 \times 10^{5}$ $\mathrm{TCID}_{50}$ per mouse) or A/PortChalmers/1/73 ((A/PC), heterosubtypic H3N2, $5.4 \times 10^{5} \mathrm{TCID}_{50}$ per mouse). Challenged mice were monitored for 3 weeks for development of clinical symptoms (including weight loss), and animals were humanely euthanized if they lost $20 \%$ of their starting body weight.

For analysis of influenza-specific T-cell responses, naive $\mathrm{CD} 8^{+} \mathrm{OT}-\mathrm{I}$ cells were isolated from the spleens of OT-I mice (transgenic C57BL/6 line described 
by ref. ${ }^{52}$ ), using the EasySep Mouse Naive $\mathrm{CD}^{+} \mathrm{T}$-cell isolation kit (Stem Cell Technologies). OT-I cell purity of at least $90 \%$ was required for all transfers. Seven-week-old female C57BL/6 mice were purchased from ARC, and $1 \times 10^{4}$ OT-I cells in $200 \mu \mathrm{l}$ of PBS were transferred intravenously per mouse. At $24 \mathrm{~h}$ post transfer, recipients were anaesthetized as above, and then vaccinated i.n. with either $\gamma$-Flu-OVA alone $\left(3 \times 10^{5} \mathrm{TCID}_{50}\right.$ equivalent per mouse in $\left.50 \mu \mathrm{l}\right)$ or $\gamma$-Flu-OVA $+\gamma$-PN $(\Delta$ PsaA $)\left(3 \times 10^{5}\right.$ TCID $_{50}$ equivalent $+10^{8} \mathrm{CFU}$ equivalent $\gamma$ - $\mathrm{PN}(\Delta \mathrm{PsaA})$ in $50 \mu \mathrm{l})$. Inactivated vaccine components were mixed and incubated on ice for $\sim 15 \mathrm{~min}$ before immunization. Control animals received PBS alone. Blood samples were collected from all mice via submandibular bleeding on days 7 and 14 post-vaccination. Mice were then euthanized on day 21 post-vaccination by $\mathrm{CO}_{2}$ inhalation, and blood, lungs, spleen and $\mathrm{mLN}$ were collected for analysis by flow cytometry.

In vitro neutralization assay. Tissue-culture plates (96-well) were seeded with $6 \times 10^{4} \mathrm{MDCK}$ cells per well. Live A/PR8 or A/Cal virus was diluted in allantoic fluid, and activated by treatment with $4 \mu \mathrm{g} \mathrm{ml}^{-1} \mathrm{~N}$-tosyl-L-phenylalanine chloromethyl ketone (TPCK)-trypsin (Sigma-Aldrich) for $30 \mathrm{~min}$ at $37^{\circ} \mathrm{C}$. Serum samples from vaccinated and control BALB/c mice were heat-inactivated for 30 min at $56^{\circ} \mathrm{C}$. Heat-inactivated serum was then serially diluted in PBS, mixed with activated IAV in a $1: 1$ ratio, and incubated for $1 \mathrm{~h}$ at $37^{\circ} \mathrm{C}$ to allow binding. $\mathrm{IAV}+$ serum mixtures were then added to MDCK monolayers at a multiplicity of infection (MOI) of 0.1 , and incubated for $2 \mathrm{~h}$ at $37^{\circ} \mathrm{C}+5 \% \mathrm{CO}_{2}$. Monolayers were then washed with PBS to remove unbound virus, and incubated for an additional $22 \mathrm{~h}$ in serum-free medium. Monolayers were then fixed for $15 \mathrm{~min}$ in ice-cold acetone/methanol (mixed in 1:1 ratio), and stained using polyclonal murine anti-A/PR8 or -A/Cal sera at 1:200 dilution (generated as previously described ${ }^{53}$ ) for $1 \mathrm{~h}$ at $4^{\circ} \mathrm{C}$. Secondary antibody Alexa-Fluor488-conjugated goat anti-mouse IgG $(\mathrm{H}+\mathrm{L})\left(1: 200\right.$ dilution, Life Technologies) was then added for $1 \mathrm{~h}$ at $4^{\circ} \mathrm{C}$, and nuclei were stained with $4^{\prime}$,6-diamidino-2-phenylindole (DAPI; $1 \mu \mathrm{g} \mathrm{ml}^{-1}$ in $\mathrm{milliQ}$ ) for $30 \mathrm{~min}$ at room temperature. Fluorescence imaging was performed using a Nikon TiE inverted fluorescence microscope, and images were analysed using NIS elements software.

Preparation of cell suspensions for flow cytometry. Mice were euthanized by $\mathrm{CO}_{2}$ asphyxiation, and $0.5 \mathrm{ml}$ of blood was immediately collected into $10 \mathrm{ml}$ PBS via cardiac puncture. Mice were then perfused with $10 \mathrm{ml}$ cold PBS through the right ventricle. Lungs were finely macerated in $1 \mathrm{ml}$ pre-warmed digestion medium (DMEM (Gibco) supplemented with 5\% FCS, $10 \mathrm{mM}$ HEPES, $2.5 \mathrm{mM} \mathrm{CaCl}_{2}$, $0.2 \mathrm{U} \mathrm{ml}^{-1}$ penicillin/gentamicin, $1 \mathrm{mg} \mathrm{ml}^{-1}$ collagenase IA (Sigma-Aldrich) and $30 \mathrm{U} \mathrm{ml}^{-1} \mathrm{DNase}$ (Sigma-Aldrich)) and incubated at $37^{\circ} \mathrm{C}$ for $1 \mathrm{~h}$, with mixing every $20 \mathrm{~min}$. Single-cell suspensions were filtered through $70 \mu \mathrm{m}$ filters (BD). Spleen and $\mathrm{mLN}$ were collected, and single-cell suspensions were prepared by mechanical disruption through a $70 \mu \mathrm{m}$ filter. All tissue samples were incubated in red cell lysis buffer $\left(155 \mathrm{mM} \mathrm{NH}_{4} \mathrm{Cl}\right.$ and $170 \mathrm{mM}$ Tris- $\mathrm{HCl}(\mathrm{pH} 7.65)$ combined at a 9:1 ratio, with $\mathrm{pH}$ adjusted to 7.2 ) for $5 \mathrm{~min}$ at $37^{\circ} \mathrm{C}$. Samples were thoroughly washed in PBS and kept on ice until staining.

Cell staining and flow cytometric analysis. Single-cell suspensions were pelleted in 96-well U-bottom trays ( $400 \mathrm{rcf}, 2 \mathrm{~min})$ at $2 \times 10^{6}$ cells per well. Cells were resuspended in near-infrared fixable dye (1:1,000 dilution in PBS, BD) for $15 \mathrm{~min}$ at room temperature in the dark. All subsequent incubations were performed at $4^{\circ} \mathrm{C}$. Cells were washed twice in FACS buffer (PBS $+1 \%$ BSA $+0.04 \%$ sodium azide), and blocked with murine $\gamma$-globulin ( $200 \mu \mathrm{g} \mathrm{ml}^{-1}$ in FACs buffer) for $10 \mathrm{~min}$. Cells were stained with the primary antibodies detailed in Supplementary Table 2 for 20-30 min. For intracellular cytokine staining, cells were first stimulated for $4 \mathrm{~h}$ with SIINFEKL peptide in restimulation medium (IMDM (Gibco) supplemented with $10 \%$ FCS, $1 \times$ penicillin/streptomycin (Gibco), $1 \times$ Glutamax (Gibco), $54 \mathrm{pM} \beta$-mercaptoethanol (Sigma), $1 \mathrm{nM}$ ionomycin (Life Technologies), GolgiStop (BD, 1/1,500 dilution of stock) and $1 \mu \mathrm{g} \mathrm{ml}^{-1}$ SIINFEKL (InVivoGen)). Stimulated cells were then washed twice in PBS before viability staining and incubation with primary antibodies against surface antigens as above. Cells were then permeabilized in BD CytoFix/CytoPerm (BD) for $20 \mathrm{~min}$. Cells were washed in Permwash (BD) and stained with a cocktail of antibodies against intracellular cytokines as per Supplementary Table 2 for $20 \mathrm{~min}$. Cells were then washed in Permwash (BD), followed by a PBS $+0.04 \%$ sodium azide wash before resuspension in $1 \%$ PFA. Data acquisition for all samples was performed on a BD LSRFortessa X-20 flow cytometer.

Focus-forming assay. Tissue-culture plates (96-well) were seeded with $6 \times 10^{4}$ MDCK cells per well. Live A/PR8 was diluted in allantoic fluid and activated by treatment with $4 \mu \mathrm{g} \mathrm{ml}^{-1} \mathrm{TPCK}$-trypsin (Sigma-Aldrich) for $30 \mathrm{~min}$ at $37^{\circ} \mathrm{C}$. $\gamma$ - $\mathrm{PN}\left(\triangle \mathrm{PsaA}\right.$ ) was serially diluted in PBS (ranging from $10^{0}-10^{7} \mathrm{CFU}$ equivalent per well of inactivated pneumococci), and mixed in a 1:1 ratio with activated A/PR8. After thorough mixing, suspensions were incubated at $37^{\circ} \mathrm{C}$ for $30 \mathrm{~min}$ and then added to MDCK monolayers to give either 100 focus-forming unit (FFU) per well of A/PR8, or A/PR8 MOI of 0.1. Cell monolayers were incubated with the virus + pneumococci mixtures for $2 \mathrm{~h}$ at $37^{\circ} \mathrm{C}$ to allow viral adhesion. Inoculum was then removed, and monolayers were washed with PBS to remove unbound virus. Monolayers were incubated for an additional $22 \mathrm{~h}$ in serum-free media, and then washed, fixed and permeabilized with acetone/methanol, and stained for IAV infection as per in vitro neutralization assay.

Macrophage uptake assay. THP-1 cells (Sigma) were maintained in RPMI $+1 \%$ penicillin/streptomycin, $1 \%$ L-glutamine, $10 \%$ FCS. Cells were seeded into 12 -well plates at $5 \times 10^{5}$ cells per well, and differentiated into macrophage-like cells with $50 \mathrm{ng} \mathrm{ml}^{-1}$ PMA for 3 days. Medium was then aspirated to remove non-adherent cells and replaced with fresh medium (no PMA). Cells were rested for $36 \mathrm{~h}$ before use in assays. For assessment of antigen uptake, live and irradiated A/PR8 was diluted to $10^{8} \mathrm{TCID}_{50} \mathrm{ml}^{-1}$ equivalent in RPMI, and mixed with $5 \times 10^{7} \mathrm{CFU} \mathrm{ml}{ }^{-1}$ equivalent $\gamma-\mathrm{PN}(\triangle \mathrm{PsaA})$ where appropriate. Suspensions were statically incubated at $37^{\circ} \mathrm{C}$ for $30 \mathrm{~min}$, and then added to washed THP- 1 monolayers ( $1 \mathrm{ml}$ diluted antigen mixture per well). Monolayers were incubated with vaccine antigens for $3 \mathrm{~h}$, and then washed thoroughly with PBS. Alternatively, cells were exposed to $\gamma-\mathrm{PN}(\Delta \mathrm{PsaA})$ alone for $1 \mathrm{~h}$, followed by extensive washing, and subsequent exposure to $\gamma$-Flu alone for $3 \mathrm{~h}$. Cells were then trypsinized for $10 \mathrm{~min}$. RPMI $+10 \%$ FCS was added to neutralize trypsin, and cells were washed thoroughly in PBS before cell counting and plating in 96-well trays at 80,000 cells per well. Cells were incubated with near-infrared fixable dye (1:1,000 dilution in PBS, BD), and Fc receptors were blocked with human sera (1:50 dilution in $\mathrm{PBS}+1 \% \mathrm{BSA}+0.04 \%$ sodium azide). Cells were permeabilized using the BD CytoFix/CytoPerm Fixation/permeabilization Solution Kit according to the manufacturer's instructions, and stained for intracellular antigen with FITCconjugated mouse anti-IAV NP (ab20921, diluted 1:150 in BD PermWash) for $30 \mathrm{~min}$ on ice. Cells were washed, and resuspended in 1\% PFA for acquisition on a BD LSRFortessa X-20 flow cytometer.

Flow cytometry to assess interaction of vaccine components. All buffers were $0.2 \mu \mathrm{m}$ filter-sterilized before use. $\gamma$ - $\mathrm{PN}(\Delta \mathrm{PsaA})$ was diluted to $5 \times 10^{8} \mathrm{CFU}$ equivalent $\mathrm{ml}^{-1}$ in sterile PBS. Increasing amounts of $\gamma$-Flu were added to diluted $\gamma$-PN $(\triangle \mathrm{PsaA})$ suspensions, ranging from $2 \times 10^{5}$ to $5 \times 10^{7} \mathrm{TCID}_{50}$ equivalent $\mathrm{ml}^{-1}$. As a control, $\gamma-\mathrm{PN}(\Delta \mathrm{PsaA})$ was also mixed with increasing amounts of allantoic fluid alone. Live and irradiated samples of $\mathrm{PN}(\triangle \mathrm{PsaA})\left(\right.$ at $5 \times 10^{8} \mathrm{CFU}$ equivalent $\mathrm{ml}^{-1}$ ) were also mixed with live or irradiated IAV (at $1 \times 10^{7} \mathrm{TCID}_{50}$ $\mathrm{ml}^{-1}$ ), or allantoic fluid or PBS alone. All mixtures were incubated for $30 \mathrm{~min}$ (unless otherwise indicated) at $37^{\circ} \mathrm{C}+5 \% \mathrm{CO}_{2}$, then spun at $10,000 \mathrm{~g}$ for $3 \mathrm{~min}$ (to pellet pneumococci but not free virions), and pellets were washed $3 \times$ in sterile PBS. Mixtures were then plated in a 96-well U-bottom tray at $5 \times 10^{7} \mathrm{CFU}$ equivalent per well, and centrifuged at $4,000 \mathrm{~g}$ for $10 \mathrm{~min}$. Pellets were then resuspended in $50 \mu \mathrm{l}$ polyclonal murine anti-A/PR8 sera diluted 1:200 in PBS $+1 \%$ BSA (generated as described in ref. ${ }^{53}$ ), and incubated on ice for $45 \mathrm{~min}$. Cells were then washed $3 \times$ in PBS, and resuspended in $50 \mu \mathrm{l}$ goat anti-mouse $\mathrm{IgG}(\mathrm{H}+\mathrm{L})$ (AlexaFluor488 conjugated, Life Technologies) diluted 1:500 in PBS + 1\% BSA. Plates were incubated on ice for $45 \mathrm{~min}$, and cells were washed $3 \times$ in PBS, then resuspended in $200 \mu \mathrm{l} 2 \%$ PFA for data acquisition on an Accuri flow cytometer. A minimum of 10,000 events were acquired per sample.

TEM. $\gamma$-Flu-OVA and $\gamma$-PN $(\Delta \mathrm{PsaA})$ preparations were mixed in $0.2 \mu \mathrm{m}$ filtersterilized PBS $\left(3 \times 10^{5} \mathrm{TCID}_{50}\right.$ equivalent $\gamma$-Flu-OVA added to $10^{8} \mathrm{CFU}$ equivalent $\gamma$-PN $(\triangle \mathrm{PsaA}))$, and incubated for $30 \mathrm{~min}$ on ice. Mixtures were then washed twice in PBS by spinning at $10,000 \mathrm{~g}$ for $3 \mathrm{~min}$. Pellets were resuspended in PBS and loaded into $3 \mathrm{~mm}$ Formvar-amorphous carbon-coated copper grids and left for $2 \mathrm{~min}$. Excess solution was removed by blotting with Whatman paper. Samples were stained with $2 \%$ uranyl acetate for $2 \mathrm{~min}$, and then blotted and left to dry at room temperature for $10 \mathrm{~min}$ before visualization with an FEI Tecnai G2 Spirit Transmission Electron Microscope (Adelaide Microscopy, University of Adelaide).

Cytokine analysis. BALB/c mice were anaesthetized and vaccinated i.n. with $\gamma$-Flu alone $\left(6.4 \times 10^{6} \mathrm{TCID}_{50}\right.$ equivalent per mouse in $\left.32 \mu \mathrm{l}\right)$, or $\gamma$-Flu + $\gamma$-PN $(\Delta \mathrm{PsaA})\left(6.4 \times 10^{6} \mathrm{TCID}_{50}\right.$ equivalent $+10^{8} \mathrm{CFU}$ equivalent $\gamma$ - $\mathrm{PN}(\Delta \mathrm{PsaA})$ in $32 \mu \mathrm{l})$. Control animals received $\gamma-\mathrm{PN}(\Delta \mathrm{PsaA})$ alone or plain PBS. On days 2,4 and 6 post-vaccination, mice were euthanized by $\mathrm{CO}_{2}$ asphyxiation, and the trachea was exposed and opened. BAL was collected by 2 sequential $0.5 \mathrm{ml}$ PBS washes of the lungs from the tracheal opening using a syringe fitted with an Insyte Autoguard catheter (BD). Custom murine Luminex immunoassay was performed by Crux Biolab.

Statistical analysis. Quantitative results are expressed as mean \pm s.e.m. For all in vivo experiments, data are representative of two independent replicate experiments with similar results, unless otherwise specified. Unpaired $t$-tests (twotailed) were used for comparison of data from two separate groups, and one-way analysis of variance (ANOVA; with Tukey's multiple comparisons test) was used for comparison of data from three or more groups. Two-way ANOVA (with Tukey's multiple comparisons test) was used when data were grouped according to two independent variables. Survival data were analysed using Fisher's exact test (twotailed). All statistical analyses were performed using GraphPad Prism 8, version 8.0.1 (GraphPad Software). $P$ values $<0.05$ (95\% confidence) were considered statistically significant. 
Ethics statement. This study was conducted in strict accordance with the Australian Code of Practice for Care and Use of Animals for Scientific Purposes (7th edition (2004), 8th edition (2013)) and the South Australian Animal Welfare Act 1985. The experimental protocols were approved by the Animal Ethics Committee at The University of Adelaide (S/2016/183 and S/2018/013).

Reporting Summary. Further information on research design is available in the Nature Research Reporting Summary linked to this article.

\section{Data availability}

The data that support the findings of this study are available from the corresponding author upon request.

Received: 10 September 2018; Accepted: 2 April 2019;

Published online: 20 May 2019

\section{References}

1. Kuss, S. K. et al. Intestinal microbiota promote enteric virus replication and systemic pathogenesis. Science 334, 249-252 (2011).

2. Kane, M. et al. Successful transmission of a retrovirus depends on the commensal microbiota. Science 334, 245-249 (2011).

3. Madhi, S. A., Klugman, K. P. \& The Vaccine Trialist Group A role for Streptococcus pneumoniae in virus-associated pneumonia. Nat. Med. 10, 811-813 (2004).

4. Johnson, P. T. \& Hoverman, J. T. Parasite diversity and coinfection determine pathogen infection success and host fitness. Proc. Natl Acad. Sci. USA 109, 9006-9011 (2012).

5. McCullers, J. A. Insights into the interaction between influenza virus and pneumococcus. Clin. Microbiol. Rev. 19, 571-582 (2006).

6. Blyth, C. C. et al. The impact of bacterial and viral co-infection in severe influenza. Influenza Other Respir. Viruses 7, 168-176 (2013).

7. Nelson, G. E., Gershman, K. A., Swerdlow, D. L., Beall, B. W. \& Moore, M. R Invasive pneumococcal disease and pandemic (H1N1) 2009, Denver, Colorado, USA. Emerg. Infect. Dis. 18, 208-216 (2012).

8. Chien, Y. W., Klugman, K. P. \& Morens, D. M. Bacterial pathogens and death during the 1918 influenza pandemic. N. Engl. J. Med. 361, 2582-2583 (2009).

9. Zhang, Y. Y. et al. Comparison of dual influenza and pneumococcal polysaccharide vaccination with influenza vaccination alone for preventing pneumonia and reducing mortality among the elderly: a meta-analysis. Hum. Vaccin. Immunother. 12, 3056-3064 (2016).

10. Chan, T. C. et al. Prevention of mortality and pneumonia among nursing home older adults by dual pneumococcal and seasonal influenza vaccination during a pandemic caused by novel pandemic influenza A (H1N1). J. Am. Med. Dir. Assoc. 13, 698-703 (2012).

11. Krammer, F. \& Palese, P. Advances in the development of influenza virus vaccines. Nat. Rev. Drug Discov. 14, 167-182 (2015).

12. Alsharifi, $M$. et al. Intranasal flu vaccine protective against seasonal and H5N1 avian influenza infections. PLoS ONE 4, e5336 (2009).

13. Furuya, Y. et al. Cytotoxic $\mathrm{T}$ cells are the predominant players providing cross-protective immunity induced by $\gamma$-irradiated influenza A viruses. J. Virol. 84, 4212-4221 (2010).

14. David, S. C. et al. The effect of gamma-irradiation conditions on the immunogenicity of whole-inactivated Influenza A virus vaccine. Vaccine 35, 1071-1079 (2017).

15. Babb, R. et al. Enhanced protective responses to a serotype-independent pneumococcal vaccine when combined with an inactivated influenza vaccine. Clin. Sci. 131, 169-180 (2017).

16. Gerlach, C. et al. The chemokine receptor CX3CR1 defines three antigenexperienced CD8 $\mathrm{T}$ cell subsets with distinct roles in immune surveillance and homeostasis. Immunity 45, 1270-1284 (2016).

17. Mueller, S. N., Gebhardt, T., Carbone, F. R. \& Heath, W. R. Memory T cell subsets, migration patterns, and tissue residence. Annu Rev. Immunol. 31, 137-161 (2013).

18. Schenkel, J. M. \& Masopust, D. Tissue-resident memory T cells. Immunity 41, 886-897 (2014).

19. Mackay, L. K. et al. The developmental pathway for $\mathrm{CD} 103^{+} \mathrm{CD} 8^{+}$tissueresident memory T cells of skin. Nat. Immunol. 14, 1294-1301 (2013).

20. Cibrian, D. \& Sanchez-Madrid, F. CD69: from activation marker to metabolic gatekeeper. Eur. J. Immunol. 47, 946-953 (2017).

21. Gunn, M. D., Nelken, N. A., Liao, X. \& Williams, L. T. Monocyte chemoattractant protein-1 is sufficient for the chemotaxis of monocytes and lymphocytes in transgenic mice but requires an additional stimulus for inflammatory activation. J. Immunol. 158, 376-383 (1997).

22. Lindell, D. M., Lane, T. E. \& Lukacs, N. W. CXCL10/CXCR3-mediated responses promote immunity to respiratory syncytial virus infection by augmenting dendritic cell and $\mathrm{CD}^{+} \mathrm{T}$ cell efficacy. Eur. J. Immunol. 38, 2168-2179 (2008)
23. Fadel, S. A., Bromley, S. K., Medoff, B. D. \& Luster, A. D. CXCR3-deficiency protects influenza-infected CCR5-deficient mice from mortality. Eur. J. Immunol. 38, 3376-3387 (2008).

24. Wu, T. et al. Lung-resident memory CD8 T cells (TRM) are indispensable for optimal cross-protection against pulmonary virus infection. J. Leukoc. Biol. 95, 215-224 (2014).

25. Zens, K. D., Chen, J. K. \& Farber, D. L. Vaccine-generated lung tissueresident memory $\mathrm{T}$ cells provide heterosubtypic protection to influenza infection. JCI Insight 1, e85832 (2016).

26. Skon, C. N. et al. Transcriptional downregulation of S1pr1 is required for the establishment of resident memory $\mathrm{CD}^{+} \mathrm{T}$ cells. Nat. Immunol. 14, 1285-1293 (2013)

27. Casey, K. A. et al. Antigen-independent differentiation and maintenance of effector-like resident memory T cells in tissues. J. Immunol. 188, 4866-4875 (2012).

28. Mueller, S. N. \& Mackay, L. K. Tissue-resident memory T cells: local specialists in immune defence. Nat. Rev. Immunol. 16, 79-89 (2016).

29. Khan, T. N., Mooster, J. L., Kilgore, A. M., Osborn, J. F. \& Nolz, J. C. Local antigen in nonlymphoid tissue promotes resident memory $\mathrm{CD}^{+} \mathrm{T}$ cell formation during viral infection. J. Exp. Med. 213, 951-966 (2016).

30. Williams, A. E., Jose, R. J., Brown, J. S. \& Chambers, R. C. Enhanced inflammation in aged mice following infection with Streptococcus pneumoniae is associated with decreased IL-10 and augmented chemokine production. Am. J. Physiol. Lung Cell Mol. Physiol. 308, L539-L549 (2015).

31. Neill, D. R. et al. Density and duration of pneumococcal carriage is maintained by transforming growth factor betal and $\mathrm{T}$ regulatory cells. Am. J. Respir. Crit. Care Med. 189, 1250-1259 (2014).

32. Slutter, B. et al. Dynamics of influenza-induced lung-resident memory T cells underlie waning heterosubtypic immunity. Sci. Immunol. 2, eaag2031 (2017).

33. Laidlaw, B. J. et al. CD4 $4^{+} \mathrm{T}$ cell help guides formation of $\mathrm{CD} 103^{+}$lungresident memory $\mathrm{CD}^{+} \mathrm{T}$ cells during influenza viral infection. Immunity 41 , 633-645 (2014)

34. Nakanishi, Y., Lu, B., Gerard, C. \& Iwasaki, A. CD8 ${ }^{+}$T lymphocyte mobilization to virus-infected tissue requires $\mathrm{CD} 4^{+} \mathrm{T}$-cell help. Nature 462 , 510-513 (2009)

35. Appay, V. et al. Characterization of $\mathrm{CD}^{+}$CTLs ex vivo. J. Immunol. 168, 5954-5958 (2002).

36. Brown, D. M., Lee, S., Garcia-Hernandez Mde, L. \& Swain, S. L. Multifunctiona $\mathrm{CD} 4$ cells expressing gamma interferon and perforin mediate protection against lethal influenza virus infection. J. Virol. 86, 6792-6803 (2012).

37. Wilkinson, T. M. et al. Preexisting influenza-specific $\mathrm{CD}^{+}{ }^{+} \mathrm{T}$ cells correlate with disease protection against influenza challenge in humans. Nat. Med. 18, 274-280 (2012).

38. Pozzi, L. A., Maciaszek, J. W. \& Rock, K. L. Both dendritic cells and macrophages can stimulate naive CD8 T cells in vivo to proliferate, develop effector function, and differentiate into memory cells. J. Immunol. 175, 2071-2081 (2005).

39. Chatziandreou, N. et al. Macrophage death following influenza vaccination initiates the inflammatory response that promotes dendritic cell function in the draining lymph node. Cell Rep. 18, 2427-2440 (2017).

40. Hoffmann, J. et al. Viral and bacterial co-infection in severe pneumonia triggers innate immune responses and specifically enhances IP-10: a translational study. Sci. Rep. 6, 38532 (2016).

41. Stark, J. M., Stark, M. A., Colasurdo, G. N. \& LeVine, A. M. Decreased bacterial clearance from the lungs of mice following primary respiratory syncytial virus infection. J. Med. Virol. 78, 829-838 (2006).

42. Alymova, I. V. et al. The novel parainfluenza virus hemagglutininneuraminidase inhibitor BCX 2798 prevents lethal synergism between a paramyxovirus and Streptococcus pneumoniae. Antimicrob. Agents Chemother. 49, 398-405 (2005).

43. Kukavica-Ibrulj, I. et al. Infection with human metapneumovirus predisposes mice to severe neumococcal pneumonia. J. Virol. 83, 1341-1349 (2009).

44. Okamoto, S. et al. The Streptococcus pyogenes capsule is required for adhesion of bacteria to virus-infected alveolar epithelial cells and lethal bacterial-viral superinfection. Infect. Immun. 72, 6068-6075 (2004).

45. Wang, Y. et al. Capsular sialic acid of Streptococcus suis serotype 2 binds to swine influenza virus and enhances bacterial interactions with virus-infected tracheal epithelial cells. Infect. Immun. 81, 4498-4508 (2013).

46. Hosaka, Y. et al. Binding of influenza type A viruses to group B Streptococcus and haemagglutination by virus-bound bacteria. J. Electron Microsc. 49, 765-773 (2000)

47. Sheffield, F. W., Smith, W. \& Belyavin, G. Purification of influenza virus by red-cell adsorption and elution. Br. J. Exp. Pathol. 35, 214-222 (1954).

48. Cottey, R., Rowe, C. A. \& Bender, B. S. Influenza Virus. Curr. Protoc. Immunol. 42, 19.11.1-19.11.32 (2001).

49. Babb, R. et al. Intranasal vaccination with gamma-irradiated Streptococcus pneumoniae whole-cell vaccine provides serotype-independent protection mediated by B-cells and innate IL-17 responses. Clin. Sci. 130, 697-710 (2016) 
50. Harvey, R. M., Ogunniyi, A. D., Chen, A. Y. \& Paton, J. C. Pneumolysin with low hemolytic activity confers an early growth advantage to Streptococcus pneumoniae in the blood. Infect. Immunol. 79, 4122-4130 (2011).

51. WHO Expert Committee on Biological Standardization (World Health Organization, 2005).

52. Hogquist, K. A. et al. T cell receptor antagonist peptides induce positive selection. Cell 76, 17-27 (1994).

53. Chan, J., Babb, R., David, S. C., McColl, S. R. \& Alsharifi, M. Vaccine-induced antibody responses prevent the induction of interferon type I responses upon a homotypic live virus challenge. Scand. J. Immunol. 83, 165-173 (2016).

\section{Acknowledgements}

The authors thank Adelaide University Microscopy (Adelaide, Australia) for assistance in sample preparation and operation of the FEI Tecnai G2 Spirit TEM. We also acknowledge the following funding sources supporting this research: an Australian Institute of Nuclear Science and Engineering (AINSE) Research Award (ALNGRA15517; to M.A.); an Australian Postgraduate Award and Gamma Vaccines Pty Ltd research sponsorship (to S.C.D.); and a National Health and Medical Research Council Senior Principal Research Fellowship (awarded to J.C.P.).

\section{Author contributions}

M.A., S.C.D. and J.C.P. conceived and designed the study. S.C.D., T.N., T.T., J.J.W., E.V.S., Z.L. and J.D. performed the experiments. S.C.D. and T.N. performed statistical analysis.
S.C.D. and M.A. wrote the manuscript. T.R.H., I.C. and S.R.M. assisted in experimental design, preparation of the manuscript and provided reagents. M.A. and J.C.P. supervised the study.

\section{Competing interests}

M.A. is head of the vaccine research group at the University of Adelaide and the Chief Scientific Officer of Gamma Vaccines Pty Ltd and Director of GPN Vaccines Pty Ltd, J.C.P. is a Director of GPN Vaccines Pty Ltd, and T.R.H. is the Executive Chairman of Gamma Vaccines Pty Ltd and GPN Vaccines Pty Ltd. This does not alter adherence to policies on sharing data and materials. Both Gamma Vaccines Pty Ltd and GPN Vaccines Pty Ltd have no role in the study design, data collection and analysis, decision to publish or preparation of the manuscript.

\section{Additional information}

Supplementary information is available for this paper at https://doi.org/10.1038/ s41564-019-0443-4.

Reprints and permissions information is available at www.nature.com/reprints. Correspondence and requests for materials should be addressed to M.A.

Publisher's note: Springer Nature remains neutral with regard to jurisdictional claims in published maps and institutional affiliations.

(c) The Author(s), under exclusive licence to Springer Nature Limited 2019 


\section{Reporting Summary}

Nature Research wishes to improve the reproducibility of the work that we publish. This form provides structure for consistency and transparency in reporting. For further information on Nature Research policies, see Authors \& Referees and the Editorial Policy Checklist.

\section{Statistical parameters}

When statistical analyses are reported, confirm that the following items are present in the relevant location (e.g. figure legend, table legend, main text, or Methods section).

$\mathrm{n} / \mathrm{a} \mid$ Confirmed

$\square$ The exact sample size $(n)$ for each experimental group/condition, given as a discrete number and unit of measurement

$\square$ \ An indication of whether measurements were taken from distinct samples or whether the same sample was measured repeatedly

$\square$ The statistical test(s) used AND whether they are one- or two-sided

Only common tests should be described solely by name; describe more complex techniques in the Methods section.

$\square$ A description of all covariates tested

$\square$ \A description of any assumptions or corrections, such as tests of normality and adjustment for multiple comparisons

$\square$ A full description of the statistics including central tendency (e.g. means) or other basic estimates (e.g. regression coefficient) AND

$\triangle$ variation (e.g. standard deviation) or associated estimates of uncertainty (e.g. confidence intervals)

$\square$ For null hypothesis testing, the test statistic (e.g. $F, t, r$ ) with confidence intervals, effect sizes, degrees of freedom and $P$ value noted

Give $P$ values as exact values whenever suitable.

Х $\square$ For Bayesian analysis, information on the choice of priors and Markov chain Monte Carlo settings

Х $\square$ For hierarchical and complex designs, identification of the appropriate level for tests and full reporting of outcomes

$\bigotimes \square$ Estimates of effect sizes (e.g. Cohen's $d$, Pearson's $r$ ), indicating how they were calculated

$\varnothing$ Clearly defined error bars

State explicitly what error bars represent (e.g. SD, SE, CI)

Our web collection on statistics for biologists may be useful.

\section{Software and code}

Policy information about availability of computer code

Data collection Flow cytometry data acquired using BD FACSDiva software and Accuri C6 software. NIS elements software used to collect data from focus forming assays and in vitro neutralisation assays.

Data analysis $\quad$ FlowJo software, version 10.4.1 used for gating and analysis of flow cytometry data. GraphPad Prism 8, version 8.0.1 (GraphPad Software, La Jolla, CA, USA) used for all quantitative data analysis.

For manuscripts utilizing custom algorithms or software that are central to the research but not yet described in published literature, software must be made available to editors/reviewers upon request. We strongly encourage code deposition in a community repository (e.g. GitHub). See the Nature Research guidelines for submitting code \& software for further information.

\section{Data}

Policy information about availability of data

All manuscripts must include a data availability statement. This statement should provide the following information, where applicable:

- Accession codes, unique identifiers, or web links for publicly available datasets

- A list of figures that have associated raw data

- A description of any restrictions on data availability

The data sets generated during and/or analysed during the current study are available from the corresponding author on reasonable request 


\section{Field-specific reporting}

Please select the best fit for your research. If you are not sure, read the appropriate sections before making your selection.

$\bigotimes$ Life sciences $\quad \square$ Behavioural \& social sciences $\square$ Ecological, evolutionary \& environmental sciences

For a reference copy of the document with all sections, see nature.com/authors/policies/ReportingSummary-flat.pdf

\section{Life sciences study design}

All studies must disclose on these points even when the disclosure is negative.

Sample size Where possible the standard deviation was determined by empirical experiments (e.g. animal weights from prior influenza experiments) and the biologically meaningful difference in mean weight that we would need to measure determined between groups (e.g. 20\% difference between control and test groups). We then derive ' $n$ ' by determining that we need to have a minimum of $80 \%$ power to detect this differences with a $p$ value of $<0.05$. Virtually all of the experiments planned are multi-parameter experiments with each parameter having a unique standard deviation profile. Therefore, for each experiment, we have based our power calculations for sample size (animal numbers) on the parameter which we find has the greatest variation (largest standard deviation). This is usually no more than $10 \%$ of the mean when $n=$ 10.

Data exclusions Exclusion criteria were established based on initial analysis of the data. If an outlier was suspected in a data set, a two-tailed Grubb's Test was performed, also called the ESD method (extreme studentized deviate). This method is recommended by Graphpad Prism v.8, and takes into account the mean and standard deviation of the data set. Any statistically significant outliers determined by this test were excluded from subsequent analysis.

$\begin{array}{lll}\text { Replication } & \text { All attempts at replication were successful. Experiments were repeated at least once and all data presented in the manuscript were }\end{array}$ successfully reproduced without exclusions. Survival data from mouse studies that is presented are combined from two independent experiments. For in vitro work, all samples were also tested in triplicate or quadruplicate in each experiment.

Randomization All mice used for experiments throughout this study were female, and all within a set age range for a given experiment (e.g. all mice were at 6-7 weeks of age at the start date of vaccination-challenge experiments). Mice were allocated randomly into experimental groups prior to any experiments commencing.

Blinding The experiments were not performed blinded. All experiments were performed by Shannon David as part of her PhD project. Staff resources for routine experimental blinding were not available.

\section{Reporting for specific materials, systems and methods}

Materials \& experimental systems

\begin{tabular}{l|l}
\hline Ia & Involved in the study \\
\hline & $\bigotimes$ Unique biological materials \\
$\square$ & $\square$ Antibodies \\
$\square$ Human research participants
\end{tabular}

\begin{tabular}{l|l}
\multicolumn{2}{l}{ Methods } \\
\hline n/a Involved in the study \\
$\square$ ChIP-seq \\
$\square$ MRI-based neuroimaging
\end{tabular}

\section{Unique biological materials}

Policy information about availability of materials

Obtaining unique materials Irradiated vaccine preparations were grown and concentrated at Adelaide University, and irradiated at ANSTO in NSW. Samples of both IAV and pneumococcal vaccines are readily available from the authors upon request.

\section{Antibodies}

Antibodies used

Below are all the commercially available antibodies used in this study. Information is as follows: target, fluorophore, supplier, clone name, catalogue number. Specific dilutions for each antibody are listed in Supplementary Table 2 of the Supplementary Material, and in the Methods Section of the main text.

Anti-CD8, BUV395, BD, clone 53-6.7, 563786 
Anti-CD44, FITC, BD, clone IM7, 553133

Anti-CD45.1, BV421, BD, clone A20, 563983

Anti-CD127, PECy7, eBioscience, clone A7R34, 14-1271-82

Anti-KLRG1, APC, eBioscience, clone 2F1, 17-5893-82

Anti-CX3CR1, PE, Biolegend, clone SA011F11, 149006

Anti-CD27, BV711, BD, clone LG.3A10, 740699

Anti-CD45.1, PE, BD, clone A20, 561872

Anti-CD44, V450, BD, clone IM7, 560452

Anti-IFN-y, PECy7, eBioscience, clone XMG1.2, 25-7311-41

Anti-GzmB, Alexa647, Biolegend, clone GB11, 515405

Anti-TNF-a, FITC, eBioscience, clone MP6-XT22, 11-7321-41

Anti-IL-17, BUV711, Biolegend, clone TC11.18H10.1, 506901

Anti-CD4, BUV395, BD, clone GK1.5, 563790

Anti-CD8, BUV737, BD, clone 53-6.7, 564297

Anti-CD45.1, FITC, BD, clone A20, 553775

Anti-CD69, PECy7, BD, clone H1.2F3, 561930

Anti-CD103, PE, eBioscience, clone 2E7, 12-1031-81

Anti-CD11a, Biotin, BD, clone HL111, 557365

Anti-CD44, V450, BD, clone IM7, 560452

Anti-Biotin, Alexa647, Biolegend, 405237

Anti-IAV Nucleoprotein, FITC, Abcam, ab20921

Anti-mouse IgG $(\mathrm{H}+\mathrm{L})$, AlexaFluor488, Life Technologies, A11001

Anti-IAV polyclonal murine antibodies used for in vitro neutralisation assays were generated in-house by i.p. immunisation of Balb/c mice with live A/PR8, A/Cal, or A/PC (as published in reference \#53).

Validation

All commercially available antibodies were validated specifically for the required experiment (flow cytometry or immunofluorescence) by the manufacturer (BD, eBioscience, Abcam, and Biolegend). Antibodies were used only on species for which they have been validated by the vendor. Validation data are available on the manufacturer's website (BD bioscience; https://www.bdbiosciences.com/eu/reagents/research/antibodies- buffers/immunology-reagents/c/744843, eBioscience; https://www.thermofisher.com/jp/en/home/life-science/cell-analysis/ flow-cytometry/antibodies-for-flow-cytometry.html). Appropriate antibody dilutions were performed based on preliminary experiments.

Reactivity of polyclonal murine antibodies was validated by immunofluorescence testing against the IAV subtype of interest, and against drifted and heterosubtypic strains to ensure no antibody cross-reactivity.

\section{Eukaryotic cell lines}

Policy information about cell lines

Cell line source(s)

THP-1 Human cell line obtained from Sigma (88081201)

Madin Darby Canine Kidney cells (MDCK) obtained from ATCC

Authentication

Cell lines were not authenticated in our laboratory.

Mycoplasma contamination

All cell lines tested negative for mycoplasma contamination.

Commonly misidentified lines (See ICLAC register)

No commonly misidentified cell lines were used.

\section{Animals and other organisms}

Policy information about studies involving animals; ARRIVE guidelines recommended for reporting animal research

Laboratory animals

Wild animals

Field-collected samples
Balb/c (wild-type), female, 6-7 weeks of age C57/BL6 (wild-type) female, 7 weeks of age

OT-I mice (C57/BL6 background) female, 6-10 weeks of age. Mice obtained from Professor Shaun McColl, University of Adelaide All mice were bred and housed at the University of Adelaide Animal Facility

No wild animals involved

No samples collected from the field 


\section{Plots}

Confirm that:

$\triangle$ The axis labels state the marker and fluorochrome used (e.g. CD4-FITC).

$\bigotimes$ The axis scales are clearly visible. Include numbers along axes only for bottom left plot of group (a 'group' is an analysis of identical markers).

$\bigotimes$ All plots are contour plots with outliers or pseudocolor plots.

$\bigotimes$ A numerical value for number of cells or percentage (with statistics) is provided.

\section{Methodology}

Sample preparation

Instrument

Software

Cell population abundance
For analysis of T-cell populations after vaccination:

C57/BL6 mice were euthanised by CO2 asphyxiation, and $0.5 \mathrm{ml}$ of blood was immediately collected into $10 \mathrm{ml}$ PBS via cardiac puncture. Mice were then perfused with $10 \mathrm{ml}$ cold PBS through the right ventricle. Lungs were finely macerated in $1 \mathrm{ml}$ prewarmed digestion medium (DMEM (Gibco) supplemented with 5\% FCS, $10 \mathrm{mM} \mathrm{HEPES,} 2.5 \mathrm{mM} \mathrm{CaCl} 2,0.2 \mathrm{U} / \mathrm{ml}$ penicillin/ gentamicin, $1 \mathrm{mg} / \mathrm{ml}$ collagenase IA (Sigma-Aldrich), and $30 \mathrm{U} / \mathrm{ml}$ DNase (Sigma-Aldrich)) and incubated at 37oC for $1 \mathrm{~h}$, with mixing every $20 \mathrm{~min}$. Single cell suspensions were filtered through 70 um filters (BD). Spleen and $\mathrm{mLN}$ were harvested, and single cell suspensions prepared by mechanical disruption through a 70 um filter. All tissue samples were incubated in red cell lysis buffer (155 mM NH4Cl and $170 \mathrm{mM}$ Tris- $\mathrm{HCl}$ (pH 7.65) combined at a 9:1 ratio, with pH adjusted to 7.2) for $5 \mathrm{~min}$ at 37oC. Samples were thoroughly washed in PBS and kept on ice until staining.

Single cell suspensions were then pelleted in 96-well U-bottom trays (400 rcf, $2 \mathrm{~min}$ ) at $2 \times 10^{\wedge} 6$ cells/well. Cells were resuspended in Near Infrared fixable dye (1:1000 dilution in PBS, BD) for $15 \mathrm{~min}$ at RT in the dark. All subsequent incubations were performed at $40 C$. Cells were washed twice in FACS buffer (PBS $+1 \%$ BSA $+0.04 \%$ Sodium Azide), and blocked with murine y-globulin (200 ug/ml in FACs buffer) for $10 \mathrm{~min}$. Cells were stained with primary antibodies detailed in Supplementary Table 2 for $20-30 \mathrm{~min}$. For intracellular cytokine staining, cells were first stimulated for $4 \mathrm{hrs}$ with SIINFEKL peptide in restimulation medium (IMDM (Gibco) supplemented with 10\% FCS, 1x penicillin/streptomycin (Gibco), 1x Glutamax (Gibco), 54 pM $\beta$ mercaptoethanol (Sigma), $1 \mathrm{nM}$ ionomycin (Life Technologies), GolgiStop (BD, 1/1500 dilution of stock) and $1 \mathrm{\mu g} / \mathrm{ml}$ SIINFEKL (InVivoGen)). Stimulated cells were then washed twice in PBS before viability staining and incubation with primary antibodies against surface antigens as above. Cells were then permeabilised in BD CytoFix/CytoPerm (BD) for 20 min. Cells washed in Permwash (BD) and stained with a cocktail of antibodies against intracellular cytokines for $20 \mathrm{~min}$. Cells were then washed in Permwash (BD), followed by a PBS $+0.04 \%$ sodium azide wash prior to resuspension in $1 \%$ PFA.

For analysis of direct binding between IAV and S. pneumoniae:

All buffers were 0.2 um filter-sterilised prior to use. $y$-PN(PsaA)was diluted to $5 \times 10^{\wedge} 8 \mathrm{CFU}$ equivalent $/ \mathrm{ml}$ in sterile PBS. Increasing amounts of $y$-Flu were added to diluted $y$-PN(PsaA) suspensions, ranging from $2 \times 10^{\wedge} 5-5 \times 10^{\wedge} 7$ TCID50 equivalent/ $\mathrm{ml}$. As a control, $y-\mathrm{PN}(\mathrm{PsaA})$ was also mixed with increasing amounts of allantoic fluid (AF) only. All mixtures were incubated for $30 \mathrm{~min}$ at $37 \mathrm{oC}+5 \% \mathrm{CO} 2$, then spun at $10,000 \mathrm{xg}$ for $3 \mathrm{~min}$ (to pellet pneumococci but not free virions), and pellets were washed in sterile PBS. Mixtures were then plated in a 96-well U-bottom tray at $5 \times 10^{\wedge} 7 \mathrm{CFU}$ equivalent/well, and centrifuged at 3,200x g for $10 \mathrm{~min}$. Pellets were then resuspended in 50 ul polyclonal murine anti-A/PR8 sera (diluted 1:200 in PBS + 1\% BSA), and incubated on ice for $45 \mathrm{~min}$. Wells were then topped up to $200 \mathrm{ul}$ in PBS, and cells washed $3 \mathrm{x}$ in PBS. Pellets then resuspended in 50 ul goat anti-mouse IgG $(\mathrm{H}+\mathrm{L})$ (AlexaFluor488 conjugated, Life Technologies) diluted 1:500 in PBS + 1\% BSA. Plates were incubated on ice for $45 \mathrm{~min}$, and cells were washed 3x in PBS, then resuspended in $200 \mathrm{ul}$ 2\% PFA for data acquisition. A minimum of 10,000 events were acquired per sample.

For analysis of IAV uptake by macrophages:

For assessment of antigen uptake, live and irradiated A/PR8 was diluted to $10^{\wedge} 6 \mathrm{TCID50} / \mathrm{ml}$ equivalent in RPMI, and mixed with $10^{\wedge} 7 \mathrm{CFU} / \mathrm{ml}$ equivalent $\mathrm{y}$-PN(PsaA) where appropriate. Suspensions were statically incubated at $370 \mathrm{C}$ for $30 \mathrm{~min}$, then added to washed THP-1 monolayers (cells seeded in 12-well trays 3 days prior. $1 \mathrm{ml}$ diluted antigen mixture added per well). Monolayers were incubated with vaccine antigens for $3 \mathrm{~h}$, then washed thoroughly with PBS. Cells were trypsinised for $10 \mathrm{~min}$. RPMl $+10 \%$ FCS was added to neutralise trypsin, and cells were washed thoroughly in PBS prior to cell counting and plating in 96-well trays at 40,000 cells/well. Cells were incubated with Near Infrared fixable dye (1:1000 dilution in PBS, BD), and Fc receptors were blocked with human sera (1:50 dilution in PBS $+1 \%$ BSA $+0.04 \%$ Sodium Azide). Cells were permeabilized using the BD CytoFix/CytoPerm Fixation/permeabilisation Solution Kit according to the manufacturer's instructions, and stained for intracellular antigen with FITC-conjugated mouse anti-IAV nucleoprotein (ab20921, diluted 1:150 in BD PermWash) for 30 min on ice. Cells were washed, and resuspended in 1\% PFA for acquisition.

Data acquisition for all murine tissues and THP-1 samples was performed on a BD LSRFortessa X-20 flow cytometer. Direct interaction of IAV and pneumococcal vaccine components was assessed using the BD Accuri C6 Plus Flow Cytometer.

All flow cytometry data was analysed using FlowJo software, v10.4.1

For isolation of CD8+ OT-I cells, spleens of OT-I mice were harvested and separated using the EasySepTM Mouse Naive CD8+ Tcell isolation kit (Stem Cell Technologies). OT-I cell purity of at least 90\% was required for all transfers to wild-type C57/BL6 mice, and was determined by flow cytometry with post-sort samples.

Following transfer and intranasal vaccination, the abundance of these transferred OT-I cells was approximately 10-20\% of all activated CD8+ T-cells in the lung at 21 days post-vaccination. Frequencies in the spleen and $\mathrm{mLN}$ were lower at the same time 
For isolation of Endogenous CD8+ T-cells and transferred OT-I cells: Transferred OT-I cells from CD45.1+ mice were identified by first gating on size and singularity, followed by exclusion of dead cells. Live cells were then gated on CD8 and CD44 to identify activated CD8+ T-cells. Expression of CD45.1 on these activated cells then allowed discrimination of endogenous CD8+ T-cells and transferred OT-I cells.

For detection of circulating memory cell subsets TCM (central memory), TPM (peripheral memory), and TEM (effector memory): Cells were gated based on size and singularity, followed by exclusion of dead cells. Live cells were then gated on CD8 and CD44 to identify activated CD8+ T-cells. CD45.1 expression allowed identification of endogenous CD8+ T-cells and transferred OT-I cells. Each population was then gated based on CD27 and CX3CR1 to identify circulating memory cell subsets TCM (CD27 +CX3CR1-), TPM (CD27+CX3CR1+), and TEM (CD27-CX3CR1+)

For detection of cytokine-positive OT-I cells: Cells were gated first on size and singularity, followed by exclusion of dead cells. Activated CD8+ T-cells were gated for based on CD8 and CD44 co-expression. Naïve CD8+ T-cells were identified by the absence of CD44. This naïve population was used to set positive gates for expression of the cytokines GzmB, TNF-a, IL-17, and IFN-y. Activated CD8+ T-cells were divided into endogenous CD8+ T-cells and transferred OT-I populations using CD45.1 expression. The cytokine gates set using the naïve population were subsequently applied to activated CD8+ T-cell subsets to identify cytokine-expressing OT-I cells.

For identification of Tissue Resident Memory (TRM) cells: Cells were first gated based on size and singularity, followed by exclusion of dead cells. CD8 and CD4 expression were then used to identify CD4+ and CD8+ T-cell populations. CD44 expression was used to identify activated T-cells in each subset, and the CD8+ T-cell subset was additionally divided into endogenous CD8+ T-cells and transferred OT-I cells based on CD45.1. CD4+ TRM cells were then identified using CD69 and CD11a co-expression. CD8+ TRM (both endogenous and transferred OT-I) were identified based on CD69 and CD103 co-expression.

For detection of IAV-positive macrophages: Cells were first gated based on size and singularity, followed by exclusion of dead cells. Live cells from samples not exposed to IAV were then used to set the positive gate for IAV nucleoprotein (NP). This gate was then applied to samples exposed to live or irradiated IAV.

Tick this box to confirm that a figure exemplifying the gating strategy is provided in the Supplementary Information. 